\title{
Bioethische Diskurse zwischen Recht, Ethik und Religion. Juristische Perspektiven - Zum Einfluss der Religion in bioethischen Beratungsgremien
}

\author{
Bijan Fateh-Moghadam
}

1. Die juristische Beobachtung der Religion in bioethischen Diskursen

Der vorliegende Beitrag entfaltet eine dezidiert juristische Perspektive auf den Einfluss der Religion in den bioethischen Diskursen der Gegenwart. Die Betonung des juristischen Beobachterstandpunktes verweist auf die Unmöglichkeit einer nicht-perspektivischen Bestimmung des Verhältnisses von Recht, Ethik und Religion. ${ }^{1}$ Es macht einen Unterschied, ob dieses Verhältnis theologisch, moral-philosophisch oder juristisch beobachtet wird. ${ }^{2}$ Die Differenz liegt dabei nicht erst in inhaltlich unterschiedlichen Angeboten für die Lösung eines gemeinsamen Problems, sondern darin, dass der Einfluss der Religion auf den bioethischen Diskurs von differenten Disziplinen als je unterschiedliches Bezugsproblem konstruiert und mit disziplineigenen Werkzeugen gelöst wird. So kann die rechtliche Regulierung bioethischer Fragen Religionsgemeinschaften und Theologen vor das Problem stellen, wie sie sich zu Rechtsnormen verhalten sollen, deren Inhalt im Widerspruch zur eigenen theologischen Lehrentwicklung zu stehen scheint. Es sind dann nicht nur inhaltliche Positionen zu bioethischen Fragen, die zwischen den Weltreligionen, ih-

1 Als Erkenntnissubjekt mit absolutem „Sehepunkt“ oder Totalperspektive käme allenfalls der „eine Gott“ in Betracht, wie es in einer Formulierung von Friedrich Wilhelm Graf heißt: „Wir endlichen Geschöpfe hingegen sind unausweichlich an einen bestimmten „Sehepunkt“ gebunden und können niemals partikulare Perspektivität überwinden“, Graf, Friedrich Wilhelm (2009), Missbrauchte Götter. Zum Menschenbilderstreit in der Moderne, München, $14 \mathrm{f}$.

2 Darüber hinaus kann dieses Verhältnis selbstverständlich auch historisch, literaturwissenschaftlich, politikwissenschaftlich, ästhetisch und nicht zuletzt soziologisch beobachtet werden. 
ren Strömungen und Konfessionen äußerst unterschiedlich ausfallen, ${ }^{3}$ sondern auch die jeweilige Haltung zum diskursiven Umgang mit differenten Positionen innerhalb der eigenen Glaubensgemeinschaft sowie zwischen Glaubensgemeinschaft und staatlichen Institutionen. ${ }^{4}$

Der juristische Blick konstruiert den Einfluss von Ethik und Religion auf den biopolitischen Diskurs dagegen als spezifisch rechtliches Bezugsproblem, namentlich als Problem der rechtlichen Legitimation ethischreligiöser Begründungen im biopolitischen Diskurs: Religion in bioethischen Diskursen ist cum grano salis rechtsstaatlich bedenklich. Dies gilt jedenfalls dann, wenn partikulare religiöse Vorstellungen des Guten zur Begründung und Durchsetzung von allgemeinverbindlichen freiheitsbeschränkenden Rechtsnormen herangezogen werden sollen. ${ }^{5}$ Als Reflexionstheorien des Rechts fragen zudem Rechtstheorie, Rechtssoziologie und Rechtsphilosophie nach den Veränderungen, die sich durch den besonderen Ethikbezug der Fragen der Biopolitik auf das Recht selbst erge-

3 Vgl. dazu die Zusammenstellung von Gutachten zu den Positionen der Weltreligionen zur aktuellen Biomedizin bei Schicktanz, Silke/Tannert, Christof/Wiedemann, Peter (Hg.) (2003), Kulturelle Aspekte der Biomedizin. Bioethik, Religionen und Alltagsperspektiven, Frankfurt a. M.

4 Hierzu Voigt, Friedemann (2008), Religion und Religionsvertreter in ethischen Diskursen und Kommissionen, in: Zichy, Michael/Grimm, Herwig (Hg.), Praxis in der Ethik. Zur Methodenreflexion in der anwendungsorientierten Moralphilosophie, Berlin, New York 249-273, 257 f. Am Beispiel des römischkatholischen Lehramtes, welches in der protestantischen Kirche keine Entsprechung finde, sowie am Beispiel der Diskussion um die Möglichkeit eines „normativen Pluralismus“ innerhalb der „protestantischen Ethik“ im Zusammenhang mit Diskussionen des Nationalen Ethikrats (NER) vgl. Voigt (2008), 261 ff.

5 In diesem Sinne geht etwa das von Thomas Gutmann geleitete rechtsphilosophische Teilprojekt „Normenbegründung im pluralistischen Staat“ des Exzellenzclusters „Religion und Politik in den Kulturen der Moderne und der Vormoderne“ der Westfälischen Wilhelms-Universität Münster davon aus, dass partikulare Vorstellungen des Guten - etwa religiöser Art - als Begründungsressourcen für staatlich gesetzte Normen schlechthin gesperrt seien, Projektbeschreibung: http://www.uni-muenster.de/Religion-undPolitik/forschung/projekte/a3.html (15.1.2010). Vgl. auch Gutmann, Thomas (2008), Christliche Imprägnierung des Strafgesetzbuchs?, in: Dreier, Horst/Hilgendorf, Eric (Hg.), Kulturelle Identität als Grund und Grenze des Rechts. Akten der IVR-Tagung vom 28.-30. September 2006 in Würzburg, Stuttgart, 295-313, 304 ff. 
ben: Führt die bioethische Debatte zu einer, Ethisierung' von Recht und Politik, über die auch ein stärkerer Einfluss der (öffentlichen) Religionen auf Recht und Politik möglich wird? ${ }^{6}$ Oder setzt sich die juristische Eigenlogik im praktischen Vollzug rechtlicher Operationen letztlich auch gegen konkurrierende normative Angebote seitens Ethik und Religionen durch? ${ }^{7}$

Der nachfolgende Beitrag geht diesen Fragen in drei Schritten nach: Nach einigen Bemerkungen zum religionssoziologischen Hintergrund der Problemstellung (2.) erfolgt ein Überblick über die normativen Grundlagen der sogenannten ethischen Neutralität des Staates (3.). Der dritte Teil untersucht schließlich, was aus dem zugrundegelegten Neutralitätsliberalismus für die rechtliche Bewertung des Einflusses der Religion in bioethischen Beratungsgremien folgt (4.). Dabei kommt es entscheidend darauf an, das unübersichtliche Feld der biomedizinischen Ethikberatung differenziert zu betrachten.

2. Nach der Säkularisierung - Öffentliche Religionen in der funktional differenzierten Gesellschaft

„Sonntags ausschlafen, ist etwas anderes als die Ablehnung der Sakramente, einen Imbiß nehmen, ist etwas anderes als die Entweihung der Fastenzeit von Yom Kippur [...]." ${ }^{\text {8 }}$ Niklas Luhmann bezieht sich auf dieses Zitat des amerikanischen Rechtstheoretikers Robert M. Cover um deutlich zu machen, dass der Begriff der Säkularisierung auf einen religiösen Beobachter verweist: Säkularisierung bezeichnet die andere

6 So Albers, Marion (2003), Die Institutionalisierung von Ethik-Kommissionen: Zur Renaissance der Ethik im Recht, in: Kritische Vierteljahresschrift für Gesetzgebung und Rechtswissenschaft 86, 419-436.

7 So Fateh-Moghadam, Bijan/Atzeni, Gina (2009), Ethisch vertretbar im Sinne des Gesetzes. Zum Verhältnis von Ethik und Recht am Beispiel der Praxis von Forschungs-Ethikkommissionen, in: Vöneky, Silja u. a. (Hg.), Legitimation ethischer Entscheidungen im Recht. Interdisziplinäre Untersuchungen, Berlin, Heidelberg, 114-143 sowie Bogner, Alexander (2009), Ethisierung und die Marginalisierung der Ethik. Zur Mikropolitik des Wissens in Ethikräten, in: Soziale Welt 60, 119-137.

8 Cover, Robert M. (1983), The Supreme Court. 1982 Term. Foreword: Nomos and Narrative, in: Harvard Law Review 97, 4-68, 8. Robert M. Cover vertritt einen pluralistischen Rechtsbegriff, der stark durch die jüdische TalmudTradition geprägt ist. Seine kulturalistisch-kommunitaristische Bestimmung des 
Seite der Religion, und es ist vor allem die Religion, die beobachtet, dass bestimmte gesellschaftliche Praxen nicht mehr religiös determiniert werden, sondern durch andere gesellschaftliche Subsysteme, etwa das Recht. ${ }^{9}$ Für alle anderen Teilsysteme der Gesellschaft wie Recht, Wirtschaft und Politik, die in der Umwelt der Religion operieren, sind die eigenen Operationen indes gerade nicht dadurch bestimmt, dass sie in der Umwelt des Religionssystems erfolgen. ${ }^{10}$ Mit anderen Worten: Das Recht bearbeitet und entscheidet selbst konstruierte Rechtsfragen gemäß seiner eigenen Funktionslogik, ohne zu fragen, zu welchen Irritationen dies bei religiösen Beobachtern führen könnte. Aus der Perspektive des Rechts kommt dem Begriff der Säkularisierung daher nur insoweit Bedeutung zu, als er mit der Ausdifferenzierung von Recht, Religion und Politik ${ }^{11}$ eine historische Bedingung der eigenen Möglichkeit bezeichnet. ${ }^{12}$ Gerade in der biopolitischen Debatte wird jedoch häufig betont, dass sich viele moderne Institutionen der deutschen Rechtsordnung, ungeachtet des beschriebenen Ausdifferenzierungsprozesses, bei historischer Betrachtung auf christlich-theologische Ursprünge zurückführen ließen. Selbst dort wo dies zutrifft, etwa bei zentralen Elementen der Verbrechenslehre im Strafrecht, folgt daraus indes nicht, dass die Interpretation des geltenden Rechts durch seine historische Genese immer schon (oder noch) theologisch infiziert wäre. ${ }^{13}$ Die Auslegung und Anwendung des positiven Rechts folgt vielmehr eigenen, rechtsordnungsspezifischen methodischen Regeln des

nomos als eines normativen Raums, in dem plurale, gleichwertige, vom Staat unabhängige Erzählungen rechtlichen Institutionen erst ihren Sinn verleihen, unterscheidet sich von der Bestimmung des Rechts als ausdifferenziertem Funktionssystem bei Luhmann.

9 Luhmann, Niklas (2002), Die Religion der Gesellschaft, Frankfurt a. M., 282 f. mit Fn. 15.

10 Luhmann (2002), 283.

11 Vgl. dazu Hofmann, Hasso (2003), Recht, Politik und Religion, in: Juristenzeitung 58, 377-385 und Roellecke, Gerd (2004), Die Entkoppelung von Recht und Religion, in: Juristenzeitung 59, 105-110.

12 Böckenförde, Ernst-Wolfgang (2006), Die Entstehung des Staates als Vorgang der Säkularisation, in: ders. (Hg.), Recht, Staat, Freiheit. Studien zur Rechtsphilosophie, Staatstheorie und Verfassungsgeschichte, Frankfurt a. M., 92-114, insb. 108.

13 Gutmann (2008), 298 mit Hinweis auf die Unterscheidung zwischen Genesis und Geltung. 
Anschlusses juristischer Argumentation an juristische Argumentation. Ein unmittelbarer Durchgriff auf religiöse oder moralische Begründungsformen ist hierbei - jedenfalls in der westlichen Rechtstradition - gerade ausgeschlossen. So wie gemäß der Lesart Max Webers im Kapitalismus der diesen erst ermöglichende protestantische „Geist der Askese" aus den stahlharten Gehäusen der Sorge um die äußeren Güter entwichen ist, ${ }^{14}$ wurden die theologischen Gehalte des Rechts in der modernen, funktional differenzierten Gesellschaft auf säkulare, genauer: rechtsinterne Begründungsformen umgestellt. ${ }^{15}$ Genau dies meint schließlich auch der „Primärbegriff der Säkularisation“, nämlich den „Entzug oder die Entlassung einer Sache, eines Territoriums oder einer Institution aus kirchlich-geistlicher Observanz und Herrschaft." "16

Säkularisation bezeichnet dann im Kern aber nichts anderes als funktionale Differenzierung. ${ }^{17}$ Säkularisierung ist ,als Prozess der funktionalen Ausdifferenzierung verschiedener funktionaler Teilsysteme oder Sphären moderner Gesellschaften“ zu verstehen, wie es nunmehr auch bei José Casanova heißt. ${ }^{18}$ An diesem Kern der Säku-

14 Weber, Max/Kaesler, Dirk (2004), Die protestantische Ethik und der Geist des Kapitalismus, München, 200 f.

15 Dass es sich bei den in der höchstrichterlichen Rechtsprechung sehr seltenen Bezugnahmen auf die „christlich-abendländische Tradition“ nur noch um Gespenster ehemals tatsächlich religiös-theologisch determinierter Rechtsinstitutionen handelt, wird letztlich auch in der Entscheidung des BVerfG zur Ladenöffnung an Adventssonntagen sichtbar: Auch hier wird unter B.II.3. betont, dass sich der Schutz des Art. 140 GG in Verbindung mit Art. 139 WRV nicht auf einen religiösen oder weltanschaulichen Sinngehalt der Sonn- und Feiertage beschränke. Die Regelung ziele in der ,säkularisierten Gesellschafts- und Staatsordnung“ (...) auch auf die Verfolgung profaner Ziele wie die der persönlichen Ruhe, Besinnung, Erholung und Zerstreuung, siehe BVerfG, 1 BvR 2857/07 vom 1.12.2009, Absatz-Nr. 154. Unter Anerkennung seiner religiös-christlichen historischen Wurzeln wird der Schutzauftrag von Art. 139 WRV i. V. m. Art. 140 GG damit als Bestandteil des positiven Verfassungsrechts zu einem sozialen Schutzauftrag umgedeutet. Die grundrechtsdogmatische Begründung des Urteils wirft allerdings im Einzelnen zahlreiche Fragen auf, die in der Staatsrechtswissenschaft präsumtiv kontroverse Diskussionen auslösen werden.

16 Lübbe, Hermann (2003), Säkularisierung. Geschichte eines ideenpolitischen Begriffs, 3. Auflage, Freiburg, 23.

17 Luhmann (2002), 125 f.: „Der fragwürdige und umstrittene Begriff der ,Säkularisierung kann somit durch funktionale Differenzierung definiert werden.“

18 Casanova, José (2008), Public Religions Revisited, in: Große Kracht, Hermann- 
larisierungsthese lässt sich ungeachtet der anhaltenden Kritik, der sie ausgesetzt ist,$^{19}$ festhalten, wenn man mit José Casanova davon ausgeht, dass die Rede von der Säkularisierung aus drei ganz verschiedenen, ungleichartigen und kein Ganzes bildenden Behauptungen besteht: ${ }^{20}$ Erstens Säkularisierung als funktionale Differenzierung, zweitens Säkularisierung als fortschreitender Niedergang religiöser Überzeugungen und drittens Säkularisierung im Sinne einer Beschränkung der Religion auf den Privatbereich. Begründete Kritik richtet sich vor allem gegen die letzten beiden Behauptungen, nicht aber gegen das Primat funktionaler Differenzierung, das für die Beschreibung moderner Gesellschaften geradezu als alternativlos erscheint. Da die Theorie funktionaler Differenzierung die Religion als eigenständiges Funktionssystem betrachtet und ihr damit einen Platz und zudem eine exklusive Aufgabe zuweist, ${ }^{21}$ steht sie gerade nicht für die Behauptung

Josef (Hg.), Christentum und Solidarität. Bestandsaufnahmen zu Sozialethik und Religionssoziologie, Paderborn, 313-338, 316. In früheren Arbeiten formuliert Casanova noch deutlich allgemeiner „die Ablösung und Emanzipation weltlicher Bereiche von religiösen Einrichtungen und Normen“"bzw. Säkularisierung als „Ausdifferenzierung von religiöser und weltlicher Sphäre“, siehe Casanova, José (2004), Religion und Öffentlichkeit. Ein Ost-/Westvergleich, in: Gabriel, Karl/Reuter, Hans-Richard (Hg.), Religion und Gesellschaft. Texte zur Religionssoziologie, Paderborn, 271-293, 273. Die gleichzeitige Verwendung der Begriffe Teilsysteme und Sphären deutet bereits darauf hin, dass der Begriff der funktionalen Differenzierung bei Casanova äußerst unscharf verwendet wird, weshalb die nachfolgenden Ausführungen sich an der klassischen systemtheoretischen Begriffsbestimmung orientieren. Vgl. hierzu Luhmann, Niklas (1998), Die Gesellschaft der Gesellschaft, 2 Bde, Frankfurt a. M., 595 ff. und dort insb. 707-776. Zur Kritik an Casanova vgl. auch Pollack, Detlef (2009), Rückkehr des Religiösen? Studien zum religiösen Wandel in Deutschland und Europa II, Tübingen, 4 ff. und 24 ff. mit Fn. 9.

19 Vgl. zusammenfassend die Beiträge in: After Secularisation (2006), The Hedgehog Review 8, zur Kritik der Kritik an der Säkularisierungsthese vgl. Pollack (2009).

20 Casanova, José (2006), Secularization Revisited: A Reply to Talal Asad, in: Scott, David/Hirschkind, Charles (Hg.), Powers of the Secular Modern: Talal Asad and His Interlocutors, Stanford, CA, 12-30, 12 f.; Casanova (2004), 272.

21 Religion garantiere die Bestimmbarkeit allen Sinnes gegen die miterlebte Verweisung ins Unbestimmbare und könne so höhere Ansprüche an Sinnvergewisserung auch in einem ,säkularisierten“ gesellschaftlichen Kontext noch befriedigen, siehe Luhmann (2002), 127-129. 
eines notwendigen Zusammenhangs von Säkularisierung bzw. Modernisierung und dem generellen Niedergang religiöser Überzeugungen und Verhaltensformen. Der „Begriff der Säkularisierung führt den, der ihn trotz allem benutzt, nicht zu der Hypothese, Religion habe in der Gesellschaft an Bedeutung verloren. “22 Die von Friedrich Wilhelm Graf eindrucksvoll diagnostizierte „Wiederkehr der Götter“, auch und gerade in sehr weitgehend modernisierten Gesellschaften wie den USA, ${ }^{23}$ scheint diese Niedergangs-Annahme zumindest bis auf weiteres auch empirisch zu widerlegen. ${ }^{24}$ Quantitative empirische Daten, die einen Bedeutungsrückgang von Formen der traditionellen Religiosität und Kirchlichkeit in Europa belegen, ${ }^{25}$ stehen dem nicht entgegen. Denn die Diagnose einer andauernden weltweiten Bedeutung des religiösen Feldes $^{26}$ in religiös pluralistischen Gesellschaften ist gerade nicht an quantitative Parameter wie die Kirchgangshäufigkeit, die Konfessionszugehörigkeit oder an Angaben über den Glauben an Gott gebunden. Die Bedeutung von Religion in modernen Gesellschaften wird an Phänomenen sichtbar, die sich eher qualitativ erfassen lassen, von denen hier nur zwei genannt seien: Zum einen in höchstrichterlichen Auseinandersetzungen mit Religionsbezug, die grundlegende Fragen der Verfassung religiös pluraler Gesellschaften verhandeln und regelmäßig eine starke Aufmerksamkeit der Medien auf sich ziehen. ${ }^{27}$ Zum anderen

22 Luhmann (2002), 284. Vor diesem Hintergrund überrascht es, dass Detlef Pollack die These der Vereinbarkeit von Religion und Moderne als einen generellen Angriff auf die Säkularisierungstheorie zu verstehen scheint, siehe Pollack (2009), 7.

23 Vgl. dazu auch Hochgeschwender, Michael (2007), Amerikanische Religion. Evangelikalismus, Pfingstlertum und Fundamentalismus, Frankfurt a. M.

24 Graf, Friedrich Wilhelm (2005), Die Wiederkehr der Götter. Religion in der modernen Kultur, München, 55 ff.

25 Pollack (2009), 79-100.

26 Bourdieu, Pierre (2009), Religion, Konstanz, $30 \mathrm{ff}$.

27 Vgl. aus der Rechtsprechung des Bundesverfassungsgerichts die Entscheidungen zum Kruzifix in der Schule (BVerfGE 93, 1 ff.), zum Körperschaftsstatus der Zeugen Jehovas (BVerfGE 102, 370), zum Schächtverbot (BVerfGE 104, 337), zu Sektenwarnungen (BVerfGE 105, 279), zum islamischen Kopftuch der Lehrerin (BVerfGE 108, 282) sowie zuletzt zum Sonntagschutz (BVerfG, 1 BvR 2857/07 vom 1.12.2009). Die Dynamik auf dem Gebiet des Religionsverfassungsrechts fasst Christian Walter in dem Satz zusammen, dass das Staatskirchenrecht des 21. Jahrhunderts nicht mehr das der insoweit ruhigen 1980er und 1990er-Jahren sein werde, siehe Walter, Christian (2008), Religiöse Freiheit 
nehmen die akademischen Deutungsversuche des Verhältnisses von Religion und Politik zu, wie nicht zuletzt die Einrichtung von interdisziplinären Forschungsverbünden zeigt, ${ }^{28}$ die sich mit der Stellung der Religion in modernen Gesellschaften befassen. ${ }^{29}$

Die zweite Behauptung, die mit der Säkularisierungstheorie verbunden wird, die Doppelthese der Abdrängung der Religion in die Privatsphäre ${ }^{30}$ und der Individualisierung der Religion, wie sie zuletzt Ulrich Beck in seinem Essay „Der eigene Gott ${ }^{\text {“ } 31}$ vertreten hat, erscheint zunächst nicht unplausibel. So lassen sich zahlreiche Beispiele für die geradezu modische individuelle Suche nach Spiritualität anführen, ${ }^{32}$ deren empirische Dimension und Entwicklung aber wenig dokumentiert ist. ${ }^{33}$ Ebenso lässt sich aber auch die Gegentendenz einer De-Privatisierung

als Gefahr? Eine Gegenrede, in: Das Deutsche Verwaltungsblatt, 1073-1080, 1080. Die Vermehrung juristischer Konflikte mit Religionsbezug (am Beispiel der USA) betont auch Graf (2005), 53 f.

28 Wie etwa dem bereits erwähnten Exzellenzcluster „Religion und Politik in den Kulturen der Vormoderne und der Moderne“ an der Universität Münster.

29 Hierbei wird auch nicht die Deutung der Phänomene mit den Phänomenen selbst verwechselt, wie Pollack gegenüber Grafs begriffsgeschichtlichen Analysen, hierzu Graf (2005), 69 ff., kritisiert, siehe Pollack (2009), 3 mit Fn. 2. Die juristische und akademische Beschäftigung mit Religion spiegelt selbstverständlich nicht notwendig eine Zunahme der Bedeutung von Religion im Sinne von quantitativ erfassbaren Zuwächsen beim Kirchgang oder des Glaubens an Gott in der Bevölkerung wider. Sie ist vielmehr selbst Ausdruck einer gesellschaftlichen, in diesen Fällen: juristischen und akademischen Bedeutung von Religion, die sich eben nicht in den von der quantitativen empirischen Religionssoziologie erfassten Daten erschöpft, sondern vor allem mit Prozessen religiöser Pluralisierung zu tun hat, die zu produktiven Irritationen im Rechts- und Wissenschaftssystem führen. Insofern gilt, dass die gesellschaftliche Bedeutung der Religion nicht zuletzt in der Renaissance der Religionssoziologie selbst sichtbaren Ausdruck findet.

30 Luckmann, Thomas (1991), Die unsichtbare Religion, Frankfurt a. M.

31 Beck, Ulrich (2008), Der eigene Gott. Von der Friedensfähigkeit und dem Gewaltpotential der Religionen, Frankfurt a. M.

32 Dazu ausführlich Knoblauch, Hubert (2009), Populäre Religion. Auf dem Weg in eine spirituelle Gesellschaft, Frankfurt a. M.; instruktiv bereits Bourdieu (2009), 243 in seinem Vortrag zur „Auflösung des Religiösen“ aus dem Jahr 1982.

33 Nach Pollack (2009), 91 und zusammenfassend 101 hat die Modernisierung zumindest keinen negativen Effekt auf bestimmte Formen der außerkirchlichen 
und Rückkehr „öffentlicher Religionen“ beschreiben. ${ }^{34}$ Ungeachtet der Frage, ob es sich hierbei tatsächlich um eine „Rückkehr“ handelt, was voraussetzen würde, dass die Religion bereits einmal privatisiert war, wird man sich darauf einigen können, dass die organisierte Religion, in Deutschland vor allem die christlichen Kirchen, aber auch der Zentralrat der Juden und die in der Deutschen Islam Konferenz (DIK) vertretenen muslimischen Dachverbände, eine sichtbar wirkende öffentliche Kraft darstellen. ${ }^{35}$ Gerade der Bereich der Biopolitik zeigt, dass von einer (Selbst-)Beschränkung der Religion auf den Privatbereich keine Rede sein kann. Die Frage nach der Rechtfertigung religiöser Einflussnahmen auf das Recht stellt sich nur weil und soweit öffentliche Religionen den Anspruch erheben, an den politischen und rechtlichen Prozessen moderner Gesellschaften aktiv und öffentlich mitwirken zu wollen. ${ }^{36}$ Der direkte Kameraschwenk vom obersten italienischen Gerichtshof in Rom auf den Vatikan in einem Bericht der Tagesschau über den tragischen Fall der inzwischen gestorbenen italienischen Wachkomapatientin Eluana Englaro ist ein einprägsamer empirischer Beleg in Form eines medialen Bildes dafür, dass sich die katholische Kirche als konkurrierende normative Deutungsmacht zum Rechtssystem definiert und in dieser Selbstbeschreibung von den Medien ernst genommen wird. ${ }^{37}$ Der noch genauer zu analysierende Umstand, dass es in Deutschland offenbar nicht möglich ist, eine gesetzliche Ethikkommission oder einen Deutschen Ethikrat (DER) einzusetzen, ohne dabei Vertreter der christlichen Kirchen und/oder der theologischen Fakultäten zu berücksichtigen, verweist ebenfalls auf die öffentliche und politische Bedeutung der Religion. Auch hieran zeigt sich, dass der quantifizierbare

Religiosität und des New Age. Allerdings blieben die Zahlen der Anhänger auBerkirchlicher Religiosität insgesamt eher klein.

34 Casanova, José (1994), Public Religions in the Modern World, Chicago, IL; Casanova (2008).

35 Knoblauch (2009), 201 ff.; 205.

36 Große Kracht, Hermann-Josef (2004), Religionen zwischen Säkularisierung und Entprivatisierung: José Casanovas These vom Wiedererstarken öffentlicher Religionen in der späten Moderne. Einführung, in: Gabriel, Karl/Reuter, HansRichard (Hg.), Religion und Gesellschaft. Texte zur Religionssoziologie, Paderborn, 269 f., 270.

37 Sterbehilfestreit in Italien: Italien streitet über Sterbehilfe für Eluana, Tagesthemen vom 6.2.2009, http://www.tagesschau.de/multimedia/video/ video446630.html (15.12.2009). 
Rückgang an gelebter Religiosität in der Bevölkerung nicht mit der öffentlichen und politischen Bedeutung der institutionalisierten Religion korrelliert. ${ }^{38}$

Genau dieser Befund, dass man sich auch in der funktional differenzierten Gesellschaft auf das Fortbestehen öffentlicher Religionen in einer sich fortwährend säkularisierenden Umwelt einzustellen habe, liegt auch der von Jürgen Habermas eingeführten Konzeption der post-säkularen Gesellschaft zugrunde. ${ }^{39}$ Diese post-säkulare Konstellation verlangt von der Religion wie vom Recht reflexive Leistungen in dem Sinne, dass sie auf das Fortbestehen des jeweils anderen Systems zumindest epistemisch eingestellt sein müssen. ${ }^{40}$ Die reflexive Leistung des Rechtsstaats besteht in der normativen Konstruktion eines Neutralitätsliberalismus.

\section{Das Faktum des Pluralismus und die ethische Neutralität des Staates}

\section{1 Das Faktum des Pluralismus}

Die politischen Auseinandersetzungen und Rechtskonflikte im Zusammenhang mit bioethischen Fragestellungen weisen eine besondere Nä-

38 Habermas, Jürgen (2007), Die öffentliche Stimme der Religion. Säkularer Staat und Glaubenspluralismus, in: Blätter für deutsche und internationale Politik, 1441, 1443: „Unabhängig von ihrem quantitativen Gewicht können Religionsgemeinschaften einen ,Sitz im Leben moderner Gesellschaften behaupten.“

39 Habermas, Jürgen (2005), Zwischen Naturalismus und Religion. Philosophische Aufsätze, Frankfurt a. M., 146; vgl. auch Habermas (2007), 1443.

40 Habermas (2005), 143. Wobei für die Religion die größere Herausforderung der Säkularisierung darin besteht, sich auf die Bedingungen religiöser Pluralität einzustellen: ,Jede Religion muss jetzt damit rechnen, in der gesellschaftlichen Kommunikation als kontingent, als Sache einer Option behandelt zu werden“ Luhmann (2002), 313. Dies zeigt etwa die anhaltende Diskussion über die Bedeutung der Anerkennung der Religionsfreiheit durch das Zweite Vatikanische Konzil in der katholischen Theologie, vgl. dazu die Beiträge der von Karl Gabriel veranstalteten Tagung: „Wie fand der Katholizismus zur Religionsfreiheit“ vom 11.-13. 3.2009 an der Universität Münster. Den optionalen Charakter der Religion betont auch Charles Taylor, der hierin gerade den Kern des „säkularen Zeitalters" erblickt; ein Zeitalter, indem der Glaube ,auch für besonders religiöse Menschen nur eine menschliche Möglichkeit neben anderen ist", siehe Taylor, Charles (2009), Ein säkulares Zeitalter, Frankfurt a. M., 15. 
he zu ethischen Orientierungsfragen auf, für die neben dem Recht auch die (Bio-)Ethik bzw. die Moral und die Religion normative Ressourcen bereithalten. Philosophen, Moraltheologen und Religionsvertreter entdecken das neue Berufsbild des Ethik-Experten und beanspruchen Mitspracherechte für die gesellschaftsweit verbindliche Regulierung bioethischer Konfliktlagen. Diese Gemengelage sich überlagernder normativer Bewertungshorizonte, die zu einer Hyper-Materialisierung des Rechts zu führen droht, löst sich auf, wenn man Ethik/Religion, Moral und Recht mit dem Frankfurter Philosophen Rainer Forst als unterschiedliche Kontexte der Rechtfertigung begreift. ${ }^{41}$ Als Vertreter eines politischen Liberalismus setzt Forst bei der Unterscheidung zwischen dem ethisch Guten und dem moralisch Richtigen ${ }^{42}$ bzw. dem Rechten ${ }^{43}$ an. Konzeptionen des ethisch Guten, zu denen insbesondere die Religionen zählen, verweisen auf diejenigen Wertvorstellungen und substantiellen ethischen Lehren, die der Einzelne als ethische oder gläubige Person als umfassende Basis eines für ihn gelungenen Lebens betrachtet. Angesichts des Faktums des vernünftigen Pluralismus, ${ }^{44}$ also eines Pluralismus einander ausschließender, aber gleichwohl vernünftiger umfassender Lehren des Guten, können diese nur einen begrenzten Geltungsanspruch erheben: Was für mich und eventuell für meine Überzeugungs- oder Glaubensgemein-

41 Forst, Rainer (2004), Kontexte der Gerechtigkeit. Politische Philosophie jenseits von Liberalismus und Kommunitarismus, Frankfurt a. M.; Forst, Rainer (2007), Das Recht auf Rechtfertigung. Elemente einer konstruktivistischen Theorie der Gerechtigkeit, Frankfurt a. M., 100-126; sowie bereits Habermas, Jürgen (1998), Faktizität und Geltung. Beiträge zur Diskurstheorie des Rechts und des demokratischen Rechtsstaats, Frankfurt a. M., 135 ff.

42 Habermas, Jürgen (1983), Diskursethik - Notizen zu einem Begründungsprogramm, in: ders. (Hg.), Moralbewußtsein und kommunikatives Handeln, Frankfurt a. M., 53-126, 113; Forst (2007), 103 ff.; 108: „Das Wesentliche der Unterscheidung zwischen Ethik und Moral liegt darin, dass in praktischen Konflikten, die nach (ethische Kontexte transzendierenden) Normen des zwischen Menschen strikt Ge- und Verbotenen verlangen, die Rechtfertigungsschwelle angehoben wird und reziprok-allgemein ausweisbare Gründe notwendig sind.“

43 Rawls, John (2005), Politischer Liberalismus, Frankfurt a. M., 266 ff.; Rawls, John (1997), Die Idee des politischen Liberalismus. Aufsätze 1978-1989, Frankfurt a. M., $364 \mathrm{ff}$.

44 Rawls (2005), 106 ff. und 12: „Eine moderne Gesellschaft ist nicht einfach durch einen Pluralismus umfassender religiöser, philosophischer und moralischer Lehren gekennzeichnet, sondern durch einen Pluralismus zwar einander ausschließender, aber gleichwohl vernünftiger umfassender Lehren.“ 
schaft gilt, muss nicht für alle anderen Menschen gelten. Das Faktum des Pluralismus lässt sich besonders eindrücklich am Beispiel religiöser Standpunkte zu grundlegenden Fragen der Bioethik studieren: ${ }^{45}$ Lassen sich zumindest noch einige grundlegende Gemeinsamkeiten der beiden großen christlichen Kirchen in Deutschland bei der Bewertung des Status des Embryos feststellen, ${ }^{46}$ so werden die Divergenzen spätestens im Vergleich mit den in Deutschland ebenfalls verankerten Weltreligionen des Islams und des Judentums sichtbar: Während die beiden christlichen Kirchen von einer umfassenden Schutzwürdigkeit des Embryos ab dem Zeitpunkt der Kernverschmelzung ausgehen, ${ }^{47}$ beginnt die volle Schutzwürdigkeit nach den Vorstellungen des seinerseits stark fragmentierten Islams je nach vertretener Beseelungslehre erst mit dem 40., 80. oder 120. Tag. Es gibt jedoch auch islamische Positionen, die dem Beseelungszeitpunkt keine entscheidende Bedeutung beimessen. ${ }^{48}$ Nach der jüdischen Religion bzw. dem jüdischen Gesetz (Halacha) erlangt der Embryo erst mit der Geburt einen eigenständigen Personenstatus, wobei eine erhöhte Schutzwürdigkeit des Embryos bereits mit dem Zeitpunkt der Beseelung am 40. Tag eintritt. ${ }^{49}$ Weiterhin unterscheidet die Halacha regelmäBig zwischen der Schutzwürdigkeit des Embryos in vivo (im Mutterleib) und in-vitro (im Labor), was den Interessen der Stammzellforschung entschieden entgegen kommt und sich auch in einer entsprechenden Forschungspraxis in Israel niederschlägt. ${ }^{50}$

45 „Gottes Gesetz gibt es nur im Plural“, siehe Graf, Friedrich Wilhelm (2006), Moses Vermächtnis. Über göttliche und menschliche Gesetze, München, 86 ff.; 89; vgl. auch Voigt (2008) und die Beiträge in Schicktanz/Tannert/Wiedemann (Hg.) (2003).

46 Dass auch hier Unterschiede im Hinblick auf die praktischen Konsequenzen für die Frage der Zulassung von Forschung an embryonalen Stammzellen bestehen zeigt die Studie von Voigt (2008) auf.

47 Höver, Gerhard/Eibach, Ulrich (2003), Die aktuelle Biomedizin aus der Sicht der christlichen Kirchen, in: Schicktanz/Tannert/Wiedemann (Hg.), 16-55, 22.

48 Ilkilic, Ilhan (2003), Die aktuelle Biomedizin aus der Sicht des Islam, in: Schicktanz/Tannert/Wiedemann (Hg.), 56-83, 62 ff. und zusammenfassend und vergleichend Kraus, Wolfgang (2003), Ein Vergleich der christlichen, islamischen und jüdischen Perspektiven, in: Schicktanz/Tannert/Wiedemann (Hg.), 107131, 126.

49 Nordmann, Yves/Birnbaum, Michel (2003), Die aktuelle Biomedizin aus der Sicht des Judentums, in: Schicktanz/Tannert/Wiedemann (Hg.), 84-105, 97 ff.

50 Nordmann/Birnbaum (2003), 97 ff. und zusammenfassend 104. 
Da sich jede dieser religiösen Positionen ausschließlich auf spezifische autoritative theologische Quellen beruft ${ }^{51}$ die von der jeweils anderen religiösen Position nicht anerkannt werden, scheint die diskursive Auflösung dieser Differenzen im Dialog der Religionsgemeinschaften unmöglich. ${ }^{52}$ Hieran kann man sehen, dass politische und rechtliche Entscheidungen über grundlegende Fragen der Bioethik schon praktisch nicht von partikularen Vorstellungen des ethisch Guten oder religiös Gebotenen abhängig gemacht werden können, wenn man nicht eine dieser unvereinbaren religiösen Positionen zur Staatsideologie erheben möchte.

Sollen bioethische Normen mit gesellschaftsweiter Verbindlichkeit ausgestattet werden, so müssen diese also mit einem Geltungsanspruch versehen werden, der sich unabhängig von ethischen oder religiösen Partikularvorstellungen formulieren lässt. Wer einen universellen Geltungsanspruch erhebt, bewegt sich immer schon im Kontext moralischer Rechtfertigung, in dem nur verallgemeinerbare und reziprok geltende Kriterien des moralisch Richtigen bzw. des Gerechten Geltung beanspruchen können. Dieses Gebot öffentlicher Rechtfertigung (public justification) erfordert, dass Freiheitseinschränkungen in einer säkularen Sprache gerechtfertigt werden müssen, die allen Bürgern gleichermaßen zugänglich ist. ${ }^{53}$

Für die Beantwortung konkreter normativer Fragen der Bioethik lassen sich aus dem formalen Prinzip öffentlicher Rechtfertigung aber ebenfalls keine hinreichend konkreten inhaltlichen Vorgaben entnehmen. Das moralische „Recht auf Rechtfertigung“ bleibt daher auf rechtlich-politische Konkretisierung, Institutionalisierung und Interpretation in Verfahren der Rechtsetzung und Rechtsanwendung angewiesen. ${ }^{54}$ Nicht obwohl, sondern gerade weil den Fragen der Bioethik fundamentale ethische Wertkonflikte zugrunde liegen, muss die gesellschaftsweit bzw. rechtsordnungsweit verbindliche Lösung bioethischer Konflikte den Ressourcen eines positiven Rechts entnommen werden, das sich selbst seine eigene Grundlage geworden ist. Mit einer

51 Man vergleiche nur die Literaturverzeichnisse der oben genannten Gutachten in Schicktanz/Tannert/Wiedemann (Hg.) (2003).

52 Auf die Unterschiedlichkeit der religiösen Positionen in bioethischen Fragen weist auch Graf hin, vgl. Graf (2006), 86; er sieht zugleich, dass dieser Befund die „relative Autonomie weltlichen Rechts“ langfristig stärken dürfte.

53 Rawls (2005), 312 ff.; Habermas (2005), 119 ff., insb. 126 ff.

54 So Forst (2007), 198 mit Fn. 22 und 313. 
Formulierung von Jürgen Habermas: Normativ gehaltvolle Botschaften können nur in der Sprache des Rechts gesellschaftsweit zirkulieren. ${ }^{55}$

Es lässt sich nun zeigen, dass die oben summarisch dargestellten Grundlagen eines politischen Liberalismus der Struktur des Religionsverfassungsrechts ${ }^{56}$ des Grundgesetzes entsprechen.

\section{2 Die ethische Neutralität des Staates - Zum verfassungsrechtlichen Hintergrund}

Das deutsche Grundgesetz verpflichtet nicht nur zur formellinstitutionellen Trennung von Staat und Kirche, sondern auch materiell zur weltanschaulich-religiösen Neutralität des Staates. ${ }^{57}$ Das Bundesverfassungsgericht wird nicht müde, diesen Grundsatz in seinen Entscheidungen zum Religionsrecht zu wiederholen. Es leitet ihn aus einer Zusammenschau verschiedener freiheits- und gleichheitsrechtlicher Vorschriften des Religionsverfassungsrechts ab: „Das Grundgesetz legt durch Art. 4 I, Art. 3 III GG sowie durch Art. 136 I und 4 und Art. 137 I WRV in Verbindung mit Art. 140 GG dem Staat als Heimstatt

55 Habermas (1998), 78.

56 Zum Begriff und zur Abgrenzung zum traditionellen Begriff des Staatskirchenrechts vgl. die Beiträge in Heinig, Hans Michael/Walter, Christian (Hg.) (2007), Staatskirchenrecht oder Religionsverfassungsrecht? Ein begriffspolitischer Grundsatzstreit, Tübingen sowie Walter, Christian (2006), Religionsverfassungsrecht, Tübingen, $128 \mathrm{ff}$. und $494 \mathrm{ff}$.

57 Morlok, Martin (2004), Art. 4 GG, in: Dreier, Horst (Hg.), Grundgesetz. Kommentar. Präambel, Artikel 1-19, Bd. 1, Tübingen, 484-549, Rn. 146 ff.; Morlok, Martin (2008), Art. 140 GG, in: Dreier, Horst (Hg.), Grundgesetz. Kommentar. Artikel 83-146, Bd. 3, München, 1512-1669, Rn. 36 ff.; Kokott, Juliane (2009), Art. 4, in: Sachs, Michael (Hg.), Grundgesetz. Kommentar, München, 237-274, Rn. 4 f.; Jarass, Hans D./Pieroth, Bodo (2009), Grundgesetz für die Bundesrepublik Deutschland. Kommentar, 10. Auflage, München, Art. 4, Rn. 5 und umfassend Huster, Stefan (2002), Die ethische Neutralität des Staates. Eine liberale Interpretation der Verfassung, Tübingen. Zum gegenwärtigen Stand der Debatte um den Grundsatz der religiös-weltanschaulichen Neutralität in der deutschen Staatsrechtswissenschaft vgl. Heinig, Hans Michael (2009), Verschärfung der oder Abschied von der Neutralität? Zwei verfehlte Alternativen in der Debatte um den herkömmlichen Grundsatz religiös-weltanschaulicher Neutralität, in: Juristenzeitung, 1136-1140. 
aller Staatsbürger ohne Ansehen der Person weltanschaulich-religiöse Neutralität auf." ${ }^{\text {58 }}$

Biomedizinische Normen betreffen indes nicht primär das Verhältnis von Staat und Kirche oder anderen Religionsgemeinschaften, sondern das Verhältnis zwischen Staat und Bürger. Bei der rechtlichen Regulierung der Biomedizin geht es normativ um die Abgrenzung von Freiheitsbereichen in besonders grundrechtssensiblen Bereichen. Die strafrechtlichen Grenzen des Schwangerschaftsabbruchs greifen beispielsweise in das körperbezogene Selbstbestimmungsrecht der Schwangeren ein und berühren zugleich mögliche Rechtspositionen des Embryos, deren Qualität bekanntlich umstritten ist. Der Grundsatz der ethischen Neutralität, verstanden als Gebot der Begründungsneutralität, ${ }^{59}$ muss hier in den jeweils betroffenen Grundrechten selbst verankert werden: ${ }^{60}$ Niemand muss staatliche Eingriffe in seine Grundrechte hinnehmen, die allein weltanschaulich-religiös begründet sind. Schutzbereich und Schranken der Grundrechte müssen neutral interpretiert werden, ${ }^{61} \mathrm{~d}$. h. die Gründe, die zur Rechtfertigung des Grundrechtseingriffs angeführt werden, müssen auf neutralen, allgemeingültigen, nicht konfessionell oder weltanschaulich gebundenen Gesichtspunkten beruhen. ${ }^{62}$ Die staatliche Neutralitätspflicht in bioethischen Fragen betont auch das BVerfG in seinem 2. Urteil zum Schwangerschaftsabbruch wenn es ausführt, das Lebensrecht des Nasciturus gelte „unabhängig von bestimmten religiösen oder philosophischen Überzeugungen, über die der Rechtsordnung eines religiös-weltanschaulich neutralen Staates kein Urteil zusteht." ${ }^{\text {63 } 3}$ Das Neutralitätsgebot schützt also, wie Stefan Huster betont, vor Eingriffen „,aus den falschen Gründen. “64 Um gute Gründe für die Beschränkung wissenschaftlicher Forschungsvorhaben und individueller Freiheiten geht es auch in den Verhandlungen bioethischer Beratungsgremien, die ein unübersichtliches Feld bilden.

\footnotetext{
58 BVerfGE 19, 206.

59 Huster (2002), $633 \mathrm{ff}$.

60 Grundlegend Huster (2002), 652 ff.

61 Huster (2002), $653 \mathrm{ff}$.

62 So BVerfGE 24, 236, 247 f. in Bezug auf den Schutzbereich; weitergehend Huster (2002), 653.

63 BVerfGE 88, 203, 252 (2. Urteil zum Schwangerschaftsabbruch).

64 Huster (2002), 657.
} 
4. Bioethische Beratungsgremien zwischen Ethik, Religion, Politik und Recht: Hyper-Materialisierung, Ethisierung, Entdifferenzierung?

Vor dem Hintergrund der dargestellten Neutralitätspflicht des Staates überrascht es, dass Recht und Politik in biomedizinischen Kontexten selbst auf Ethik und damit möglicherweise sogar auf Religionen als umfassende Theorien des ethisch Guten zu verweisen scheinen, indem etwa medizinrechtliche Gesetze die „ethische Vertretbarkeit“ von Forschungsvorhaben zu einer gesetzlichen Voraussetzung machen, die durch interdisziplinär besetzte Ethikkommissionen zu prüfen ist. Und auch die Einsetzung des mit mehreren religiösen Akteuren besetzten DER wirft Fragen im Hinblick auf das staatliche Neutralitätsgebot auf.

Die im Folgenden zu entfaltende These besteht darin, dass es auch für die Beurteilung ethischer und religiöser Einflüsse in bioethischen Beratungsgremien darauf ankommt, zwischen unterschiedlichen Kontexten der Rechtfertigung zu differenzieren. Es können drei Ebenen der Rechtfertigung unterschieden werden: Die erste Ebene bildet die Anwendung von Rechtsnormen durch Ethikkommissionen im Rahmen von medizinrechtlichen Verwaltungsverfahren (1.). Hierzu gehören insbesondere die Zentrale Ethikkommission des Stammzellgesetzes, ${ }^{65}$ die Forschungs-Ethikkommissionen des Arzneimittelgesetzes ${ }^{66}$ und die sogenannten Lebendspende-Kommissionen des Transplantationsgesetzes. ${ }^{67}$ Die zweite Ebene betrifft neuartige Formen staatlich institutionalisierter und privilegierter Politikberatung in bioethischen Fragen, also insbesondere den $\mathrm{DER}^{68}$ (2.). Die dritte Ebene bildet die Zivilgesellschaft, in der sich vielfältige bioethische Beratungsgremien i. w. S. identifizieren lassen (3.).

$65 \int 9$ Stammzellgesetz $=$ Gesetz zur Sicherstellung des Embryonenschutzes im Zusammenhang mit Einfuhr und Verwendung menschlicher embryonaler Stammzellen (Stammzellgesetz - StZG) vom 28. 6.2002, BGBl. 2002, $2277 \mathrm{ff}$.

66 SS 40-42 Arzneimittelgesetz = Gesetz über den Verkehr mit Arzneimitteln (Arzneimittelgesetz - AMG) in der Fassung der Bekanntmachung der Neufassung des Arzneimittelgesetzes vom 12.12.2005, BGBl. 2005, 3394 ff.

67 \ 8 III Transplantationsgesetz $=$ Transplantationsgesetz in der Fassung der Bekanntmachung vom 4. 9.2007 (BGBl. I, 2206), das durch Artikel 3 des Gesetzes vom 17. 6.2009 (BGBl. I, 1990) geändert worden ist.

$68 \int 1$ Ethikratgesetz $=$ Gesetz zur Einrichtung des Deutschen Ethikrats (Ethikratgesetz - EthRG) vom 16.7.2007, BGBl. I, 1385. 


\section{1 Rechtsanwendung: Ethikkommissionen im Verwaltungsverfahren}

Den Rechtfertigungskontext mit der größten Nähe zu dem durch das Neutralitätsgebot verpflichteten Staat bildet die Ebene der Rechtsanwendung durch Ethikkommissionen, die im Rahmen von Verwaltungsverfahren den gesetzlichen Auftrag haben die „ethische Vertretbarkeit“ medizinischer Forschungsvorhaben oder Behandlungsmaßnahmen zu beurteilen. Nach dem Selbstverständnis dieser Kommissionen verweist das Recht hier selbst auf einen außerrechtlichen Bewertungsmaßstab. So besteht die Aufgabe von Forschungs-Ethikkommissionen gemäß \2 I 1 der Verordnung über die Ethikkommission des Landes Berlin darin, die ethische Vertretbarkeit und die Rechtmäßigkeit klinischer Prüfungen von Arzneimitteln bei Menschen zu bewerten. ${ }^{69}$ Nach dieser Lesart führt die Einrichtung gesetzlicher Ethikkommissionen nicht lediglich zu einer Prozeduralisierung des rechtlichen Zulassungsverfahrens, sondern zu einer Hyper-Materialisierung: Sie eröffnet einen konkurrierenden materiell-inhaltlichen Bewertungshorizont, der den rechtlichen überlagert. Im Sinne der These einer „Renaissance der Ethik im Recht“ formuliert etwa Marion Albers allgemein für Ethikkommissionen: „Ethik-Kommissionen sollen Entwicklungen und Projekte aus ethischer Sicht beurteilen; ihr Entscheidungsmaßstab stützt sich also auf die ,Ethik““. 70

Bei genauerer Betrachtung der rechtlichen Rahmenbedingungen der Ethikkommissionen lässt sich indes zeigen, dass diese Ethisierungs- und Entdifferenzierungsthese nicht überzeugen kann. ${ }^{71}$ Die rechtlichen Barrieren für religiöse und ethische Begründungen von hoheitlichem Verwaltungshandeln in grundrechtsrelevanten Bereichen bleiben undurchlässig. Im Kontext rechtlicher Rechtfertigung, in dem gesetzliche Ethikkommissionen immer schon situiert sind, lösen sich als ethisch bezeichnete Entscheidungsmaßstäbe im praktischen Vollzug der Rechtsanwendung notwendig in rechtliche Kriterien auf. Die Tätigkeit der Ethikkommissionen unterliegt den Anforderungen an die öffentliche Rechtfertigung des Verwaltungshandelns, so dass ihre Entscheidungen und Voten nicht auf religiös-weltanschauliche Gründe oder partikulare Konzeptio-

69 GVBl. für Berlin, 62. Jhg., Nr. 2, vom 20.1.2006, 26.

70 Albers (2003), 419. Vgl. aber auch die differenzierende Analyse der Autorin ebenda, $427 \mathrm{ff}$.

71 Hierzu ausführlich Fateh-Moghadam/Atzeni (2009), 119-129 und dort 129 ff. zu den empirisch-soziologischen Ergebnissen einer qualitativen Studie. 
nen des ethisch Guten gestützt werden dürfen. Allerdings macht die Besetzung dieser Kommissionen mit Ethik-Experten, Theologen und Religionsvertretern normative Aporien ihrer rechtlichen Konstruktion sichtbar und wirft Fragen nach der Zweckmäßigkeit der eingerichteten Verfahren auf.

a) Die Zentrale Ethikkommission des Stammzellgesetzes (ZES)

Die ZES ist in dieser Hinsicht die wohl bemerkenswerteste Erscheinung in der bundesdeutschen Kommissionslandschaft. Die Tragödie des normativ widersprüchlichen rechtlichen Status des Embryos in der deutschen Rechtsordnung ${ }^{72}$ wiederholt sich auf der verfahrensrechtlichen Ebene als Farce eines ethischen Beratungsverfahrens. Die Einrichtung einer prominent besetzten Zentralen Ethikkommission suggeriert, dass hier grundlegende Fragen der Bioethik verhandelt würden, die sich im Zusammenhang mit der Forschung an embryonalen Stammzellen stellen. Bei genauerem Hinsehen handelt es sich indes lediglich um ein Verfahrensmodell, indem bestimmte, rechtlich eng definierte Voraussetzungen der Forschung an embryonalen Stammzellen unter Einbeziehung interdisziplinären Sachverstandes präventiv geprüft werden. Gemäß $\int 9$ StZG prüft und bewertet die ZES, ob die Voraussetzungen nach $\int 5$ StZG erfüllt sind und das Forschungsvorhaben in diesem Sinne ethisch vertretbar ist. Die Voraussetzungen des $\int 5$ StZG beschränken sich auf eine an wissenschaftsinternen Kriterien orientierte Prüfung der Hochrangigkeit der Forschungsziele ${ }^{73}$ und der

72 Der Embryo wird in-vitro stärker geschützt als in-vivo; während das Embryonenschutzgesetz von der Annahme einer vollen (Würde-)Schutzwürdigkeit jeder befruchteten Eizelle ausgeht, ist der Embryo im Mutterleib bis zur Nidation überhaupt nicht geschützt (Spirale; „Pille danach“) und genießt sodann im Rahmen der strafrechtlichen Vorschriften zum Schwangerschaftsabbruch einen abgestuften Schutz, der insgesamt eher schwach ist, wie das Problem der Spätabtreibung zeigt; zum Ganzen vgl. Merkel, Reinhard (2007), Der Schwangerschaftsabbruch, in: Roxin, Claus/Schroth, Ulrich (Hg.), Handbuch des Medizinstrafrechts, 3. Auflage, Stuttgart, 145-231 und Schroth, Ulrich (2007), Stammzellenforschung und Präimplantationsdiagnostik aus juristischer und ethischer Sicht, in: Roxin, Claus/ders. (Hg.), Handbuch des Medizinstrafrechts, 3. Auflage, Stuttgart, 435-461.

73 Das Gesetz verwendet einen wissenschaftsinternen Begriff der Hochrangigkeit, so auch Birnbacher, Dieter (2003), „Hochrangigkeit“ im Stammzellgesetz - Ein Kommentar aus ethischer Sicht, in: Honnefelder, Ludger/Streffer, Christian (Hg.), Jahrbuch für Wissenschaft und Ethik, Bd. 8, 353-359, 356. 
Alternativlosigkeit des Einsatzes von embryonalen Stammzellen. Die eigentlichen normativen Grundentscheidungen, das generelle Verbot der Gewinnung von embryonalen Stammzellen in Deutschland und die (dynamische) Stichtagsregelung für den Import von ausländischen embryonalen Stammzelllinien, hat der Gesetzgeber selbst getroffen, wozu er aus verfassungsrechtlichen Gründen (Vorrang und Vorbehalt des Gesetzes) auch verpflichtet war. Die Ethikkommission ist auf die Prüfung eines denkbar eng formulierten rechtlichen Konditionalprogramms beschränkt: Wenn die Hochrangigkeit und die Alternativlosigkeit zu bejahen sind, dann ist der Import der Stammzellen ethisch vertretbar im Sinne des Gesetzes. ${ }^{74}$ Die ZES handelt also genau dann rechtswidrig, wenn sie das rechtlich vorgegebene Korsett verlässt und nach eigenen, genuin ethischen Kriterien entscheidet. Dies überwachen das Robert Koch-Institut (RKI) als Genehmigungsbehörde und im Konfliktfall die Verwaltungsgerichte.

Vor diesem Hintergrund stellt sich dann aber die Frage nach der Zweckmäßigkeit der gesetzlichen Zusammensetzung der ZES, die vier Sachverständige aus den Fachrichtungen Ethik und Theologie vorsieht. ${ }^{75}$ Es ist nicht leicht $\mathrm{zu}$ sehen, wodurch Moralphilosophen, akademische Theologen oder gar Vertreter der Religionsgemeinschaften für die Beurteilung der Voraussetzungen des $\ 5$ Stammzellgesetzes in besonderer Weise qualifiziert sind. ${ }^{76}$ Tatsächlich ist die ZES gegenwärtig gleich mit vier religiösen Akteuren, zwei katholischen und zwei evangelischen Theologen, besetzt. ${ }^{77}$ Wie sich die Teilnahme an einer Kommission, die die generelle Zulässigkeit der Forschung an aus Embryonen gewonnenen Stammzellen voraussetzt und die von 34 Forschungsanträgen bisher 32 genehmigt hat ${ }^{78}$ mit den jeweiligen

74 So auch Birnbacher (2003), 356: Die Rolle der Ethikkommission werde so auf eine rein symbolische Funktion reduziert.

75 Die ZES setzt sich gemäß 8 StZG aus neun Sachverständigen der Fachrichtungen Biologie, Ethik, Medizin und Theologie zusammen. Vier der Sachverständigen werden aus den Fachrichtungen Ethik und Theologie, fünf der Sachverständigen aus den Fachrichtungen Biologie und Medizin berufen.

76 Kritisch auch Birnbacher (2003), $354 \mathrm{f}$.

77 Gemäß den Angaben des RKI (Stand: 15.10.2009); einzusehen unter: http://www.rki.de/cln_160/nn_207092/DE/Content/Gesund/Stammzellen /ZES/Mitglieder/mitglieder__node.html?__nnn=true (15.1.2010).

78 Für den Zeitraum bis zum 30.11.2008 gemäß den Angaben der Tätigkeitberichte der ZES. 
christlich-theologischen Positionen zum moralischen Status des Embryos verträgt, ist eine hier nicht zu diskutierende theologische Frage. ${ }^{79}$ Mit Blick auf die oben dargestellten Positionen der Weltreligionen zum Status des Embryos würde die Besetzung der ZES mit Vertretern der jüdischen Religionsgemeinschaft aber prima facie näher liegen.

b) Die Ethikkommissionen des Arzneimittelgesetzes

Die historische Entwicklung der Forschungs-Ethikkommissionen des AMG steht exemplarisch für die Eigendynamik der Verrechtlichung von Ethikkommissionen: Die ursprünglich wissenschaftsinternen, ethischen Beratungsgremien haben sich mit der 12. AMG-Novelle zu Patientenschutzinstitutionen mit Behördencharakter entwickelt. ${ }^{80}$ Der Grundsatz der Gesetzmäßigkeit der Verwaltung fungiert hier insofern als Entdifferenzierungssperre, als die Entscheidungen der Ethikkommissionen aufgrund gesetzlicher Grundlagen erfolgen müssen, die die wesentlichen Entscheidungen über die Zulässigkeit von klinischen Prüfungen von Arzneimitteln selbst treffen. ${ }^{81}$ Die in $\ 40 \mathrm{ff}$. AMG abschließend umschriebenen rechtlichen Versagungskriterien ${ }^{82}$ für die klinische Prüfung von Arzneimitteln lassen daher zu Recht keinerlei ethischen Spielraum zu. ${ }^{83}$

Auch hier stellen sich Fragen nach der Zweckmäßigkeit der Besetzung der Kommissionen mit Ethik-Experten und religiösen Akteuren, wie sie etwa das Bayerische Ausführungsgesetz zum AMG vorsieht. Diese Position kann von Theologen, Vertretern der Religionsgemeinschaften, Philosophen, aber auch von Ärzten mit „,besonderer wissenschaftlicher

79 Dass man hier aus theologischer Sicht durchaus zu differenzierenden Ergebnisses kommen kann, zeigt Voigt (2008), $261 \mathrm{ff}$.

80 Dazu ausführlich Wölk, Florian (2002), Zwischen ethischer Beratung und rechtlicher Kontrolle - Aufgaben und Funktionswandel der Ethikkommissionen in der medizinischen Forschung am Menschen, in: Ethik in der Medizin 14, 252 269; Taupitz, Jochen (2003), Ethikkommissionen in der Politik: Bleibt die Ethik auf der Strecke?, in: Juristenzeitung, 815-821.; Deutsch, Erwin (2006), Das neue Bild der Ethikkommission, in: Medizinrecht 24, 411-416 sowie FatehMoghadam/Atzeni (2009).

81 Zur Wesentlichkeitsdoktrin des Bundesverfassungsgerichts vgl. BVerfGE 83, $130,149 \mathrm{ff}$.

82 Deutsch (2006), 415.

83 Zur rechtsstaatlichen Notwendigkeit dieser Entwicklung sowie zur Bedeutung des verwaltungsrechtlichen Instituts des Beurteilungsspielraums vgl. ausführlich Fateh-Moghadam/Atzeni (2009), 123 f. und 127 f. 
oder beruflicher Erfahrung auf dem Gebiet der Ethik in der Medizin“ besetzt werden. ${ }^{84}$ Die empirisch-soziologischen Daten zur Praxis der Kommissionen zeigen dabei, dass die Expertise der Ethik-Experten vor allem darin besteht, gerade nicht Experte zu sein, sondern als Laie mit gesundem Menschenverstand einen kritischen Blick auf das Forschungsdesign und die Aufklärungsbögen zu werfen. ${ }^{85}$ Vor diesem Hintergrund ist es konsequent und in der Sache vorzugswürdig, wenn die AMG-Ethikkommission des Landes Berlin auf die Beteiligung von Mitgliedern mit „besonderer ethischer Kompetenz" verzichtet und stattdessen, neben den Vertretern der Medizin und der Wissenschaft, schlicht mit ,zwei Laien“ zu besetzen ist. ${ }^{86}$ Bei diesen Laien kann es sich selbstverständlich auch um religiöse Akteure handeln, diese nehmen die Aufgabe dann aber nicht als solche war, sondern eben als Laien. Dadurch, dass hier der Laie zum Experten gemacht wird, entsteht dann aus Sicht der Theologie die zunächst überraschende Frage, ob und wie Theologen und andere religiöse Akteure eine besondere „Laienkompetenz" ausbilden können, die sie für die Tätigkeit in Ethikkommissionen qualifiziert. ${ }^{87}$

\section{2 Institutionalisierte Politikberatung - Der Deutsche Ethikrat}

Der zweite praktische Kontext in dem sich die Frage nach der Rechtfertigung des Einflusses von religiösen Akteuren stellt, ist die Ebene der staatlich institutionalisierten und privilegierten Politikberatung mit dem

84 So die Formulierung in Art. 29c Abs. 1 S. 3 Bayerisches Gesundheitsdienstund Verbraucherschutzgesetz (GDVG); vgl. Gesetz über den öffentlichen Gesundheits- und Veterinärdienst, die Ernährung und den Verbraucherschutz sowie die Lebensmittelüberwachung (Gesundheitsdienst- und Verbraucherschutzgesetz - GDVG) vom 24.7.2003, GVB1. 2003, 452.

85 Fateh-Moghadam/Atzeni (2009), 139 f. Für den Ethikrat kommt Bogner (2009), 129, zu sehr ähnlichen Ergebnissen: „Niemand muss nachts bei Kant und Rawls nachlesen, um am nächsten Tag in der Kommissionssitzung bestehen zu können."

86 \ 2 Abs. 2 S. 3 Nr. 6 des Gesetzes zur Errichtung einer Ethik-Kommission des Landes Berlin vom 7.9.2005, GVBl. Berlin, 61. Jhg., Nr. 32, 16.9.2005, 466. Dies hat zudem den Vorteil, dass es sich bei den Laien gerade nicht um Mediziner oder Wissenschaftler mit ethischer Zusatzqualifikation handeln darf, wie es etwa in Bayern der Fall ist, wodurch etwaige Rollenkonflikte vermieden werden.

87 Vgl. die Anregung von Schleissing, Stephan/Zichy, Michael (2009), Religion in bioethischen Diskursen. Internationale und interreligiöse Perspektiven. Rück- 
zentralen Beispiel des DER. Sie bewegt sich zwischen der unmittelbar staatlichen Ebene der Rechtsanwendung und dem allgemeinen praktischen Diskurs der Zivilgesellschaft und ist daher mit Blick auf die Anforderungen an die religiös-weltanschauliche Neutralität des Staates am schwierigsten zu beurteilen. Eine abschließende Antwort wird daher erst am Ende dieses Beitrags, nach der Erörterung der Ebene der Zivilgesellschaft, möglich sein.

Der DER ist ein durch Gesetz eingerichteter unabhängiger Sachverständigenrat zur Bewertung ethischer Fragestellungen in den Lebenswissenschaften. ${ }^{88}$ Er soll gemäß der amtlichen Begründung des Ethikratgesetzes zugleich ein nationales Forum des Dialogs über ethische Fragen in den Lebenswissenschaften sein. ${ }^{89} \mathrm{Zu}$ seinen Aufgaben gehören insbesondere: 1. Die Information der Öffentlichkeit und Förderung der Diskussion in der Gesellschaft unter Einbeziehung der verschiedenen gesellschaftlichen Gruppen (Deliberation „von oben“); 2. Die Erarbeitung von Stellungnahmen sowie von Empfehlungen für politisches und gesetzgeberisches Handeln (Politikberatung) und 3. die Zusammenarbeit mit nationalen Ethikräten und vergleichbaren Einrichtungen anderer Staaten und internationaler Organisationen (Repräsentation).

Diese Aufgaben sollen von einem interdisziplinären Gremium von Personen erfüllt werden, die zu gleichen Teilen vom Bundestag und der Bundesregierung berufen werden. Der DER besteht aus 26 Mitgliedern, die neben naturwissenschaftlichen, medizinischen, sozialen und ökonomischen auch theologische, philosophische, ethische und rechtliche Belange in besonderer Weise repräsentieren sollen. ${ }^{90}$ Dabei soll es sich einerseits um Wissenschaftler aus den genannten Wissenschaftsgebieten handeln, andererseits um ,anerkannte Personen, die in besonderer Weise mit ethischen Fragen der Lebenswissenschaften vertraut sind.“91 Das Gesetz legt Wert darauf, dass im Ethikrat unterschiedliche „ethische Ansätze" und ein plurales Meinungsspektrum vertreten sind. ${ }^{92}$ Der Ethikrat

blick auf ein Symposium vom 18. bis 19. Februar 2009 in München, in: Zeitschrift für Evangelische Ethik 3, 215-219.

$88 \int 1$ EthRG, Ethikratgesetz $=$ Gesetz zur Einrichtung des Deutschen Ethikrats (Ethikratgesetz - EthRG) vom 16.7.2007, BGBl. I, 1385.

89 Begründung zum EthRG, BT-Drucks 16/2856.

$90 \int 4$ I 1 EthRG.

$91 \int 4$ I 2 EthRG.

$92 \int 4$ II EthRG, wobei der Begriff ,ethische Ansätze“ unbestimmt bleibt. 
ist von anderen staatlichen Einrichtungen unabhängig und seine Mitglieder üben ihr Amt persönlich und unabhängig aus. ${ }^{93}$

Es handelt sich mithin um ein hybrides Gebilde aus einem institutionalisierten Gremium der Deliberation von oben, das die Meinungsvielfalt in der Gesellschaft abbilden und zugleich aktiv strukturieren soll, ${ }^{94}$ einem wissenschaftlichen Expertengremium zur Politikberatung und einer Institution, die die Bundesrepublik Deutschland in ethischen Fragen nach außen vertreten soll. Für die Frage der Legitimationsanforderungen ist von Bedeutung, dass der Ethikrat keine verbindlichen Entscheidungen trifft und die Entscheidungen von Bundesregierung und Parlament auch nicht faktisch präjudizieren soll..$^{95}$ In der verwaltungsrechtlichen Terminologie von Schmidt-Aßmann handelt es sich mithin nicht um ein Entscheidungsgremium, sondern um ein Gremium privilegierter Beratung. ${ }^{96}$ Die Anforderungen an die rechtsstaatliche Legitimation sind hier geringer als bei vergleichbaren unabhängigen Entscheidungsgremien wie der Bundesprüfstelle für jugendgefährdende Medien. Als ein staatliches Gremium privilegierter (Politik-)Beratung ist der Ethikrat aber auch nicht frei von öffentlich-rechtlichen Bindungen. So spricht viel dafür, dass die ethische Neutralitätspflicht des Staates hier zumindest in seinem objektiv-rechtlichen Gehalt in Form des Gleichbehandlungsgebots (Parität) ${ }^{97}$ aktiviert wird, das hier als Benachteiligungs- bzw. Bevorzugungsverbot wirkt. Dass der Ethikrat überhaupt mit religiösen Akteu-

$93 \int 3$ EthRG.

94 Die Idee, die Diskussion in der Gesellschaft, also gewissermaßen den ,herrschaftsfreien Diskurs“ der ,wilden politischen Öffentlichkeit“ (Habermas) über bioethische Fragen aktiv von oben zu „,fördern“ und zu steuern, entspricht dem Paradigma der „Bürgergesellschaft“, das in der politischen Soziologie als „,verdeckter Paternalismus eines politischen Programms“ diskutiert wird, vgl. Sutter, Barbara/Maasen, Sabine (2010), "Bürgergesellschaft". Der verdeckte Paternalismus eines politischen Programms, in: Fateh-Moghadam, Bijan/Sellmaier, Stephan/Vossenkuhl, Wilhelm (Hg.), Grenzen des Paternalismus, Stuttgart, 318340 .

95 Dies soll auch $\int 4$ Abs. 3 EthRG unterstützen, wonach die Mitglieder des Ethikrats weder einer gesetzgebenden Körperschaft des Bundes oder eines Landes noch der Bundesregierung oder einer Landesregierung angehören.

96 Schmidt-Aßmann, Eberhard (2001), Grundrechtspositionen und Legitimationsfragen im öffentlichen Gesundheitswesen. Verfassungsrechtliche Anforderungen an Entscheidungsgremien in der gesetzlichen Krankenversicherung und im Transplantationswesen, Berlin, New York, 79 ff.

97 Morlok (2008), Rn. 41. 
ren besetzt werden darf, ist aus Sicht des deutschen Religionsverfassungsrechts, das durch den Grundsatz der ,offenen Neutralität" geprägt ist ${ }^{98}$, nicht zu beanstanden; wenn der Staat aber Religionsgemeinschaften beteiligt, dann muss er dies gleichmäßig tun. Auch $\int 4$ II EthRG, nach dem im Ethikrat unterschiedliche ethische Ansätze und ein plurales Meinungsspektrum vertreten sein sollen, scheint auf eine Gleichbehandlung der Religionsgemeinschaften zumindest im Sinne einer abgestuften Chancengleichheit hinzuwirken. Dass die Besetzung des DER unter dem Gesichtspunkt der religiös-weltanschaulichen Neutralität gewissen verfassungsrechtlichen Grenzen unterliegen muss, wird deutlich, wenn man sich den Extremfall vorstellt, der Gesetzgeber hätte einen „Christlichen Deutschen Ethikrat" oder gar einen „Katholischen Deutschen Ethikrat" eingerichtet. Das Beispiel zeigt, dass es auch auf der Ebene staatlich institutionalisierter Politikberatung verfassungsrechtliche Schranken geben muss, die aus der ethischen Neutralität des Staates folgen. In der tatsächlichen Besetzungspraxis wurde das Neutralitätsgebot allerdings nicht beachtet. Der DER wurde mit vier eindeutig religiösen Akteuren, zwei Theologen und zwei Bischöfen besetzt, die sämtlich einer der beiden großen christlichen Kirchen bzw. Konfessionen zugeordnet werden können. Die Diskriminierung anderer Religionsgemeinschaften, insbesondere des Judentums und des Islams wirkt hier um so schwerer, als die Mitglieder des Ethikrats nicht als Repräsentanten von Organisationen, sondern als individuelle Persönlichkeiten berufen werden, so dass die fehlende Organisations- und Vertretungsstruktur der islamischen Religionsgemeinschaft von vornherein kein Problem bereitet. Die engstirnige Beschränkung auf Vertreter der christlichen Konfessionen in einem Gremium, das ethische Grundfragen verhandeln soll, führt zu einer „kontra-

98 Offene Neutralität ist eine integrierende Neutralität, die religiösweltanschauliche Äußerungen als Ausdruck der Religionsfreiheit paritätisch unterstützt, vgl. Czermak, Gerhard/Hilgendorf, Eric (2008), Religions- und Weltanschauungsrecht. Eine Einführung, Berlin, 94 f. Im Verfassungsrecht wird auch von einer ,hinkenden Trennung“"von Staat und Kirche, vgl. Jarass/Pieroth (2009), Art. 4, Rn. 4 und Art. 140/137 WRV, Rn. 2; Brugger, Winfried (2007), Von Feindschaft über Anerkennung zur Identifikation. Staat-Kirche-Modelle und ihr Verhältnis zur Religionsfreiheit, in: Joas, Hans/Wiegandt, Klaus (Hg.), Säkularisierung und die Weltreligionen, Frankfurt a. M., 253-283, 265 oder von einem Kooperationsmodell, vgl. Walter (2006), 96 ff.; Kokott (2009), Rn. 6, gesprochen. Jedenfalls beruht die religionsverfassungsrechtliche Ordnung des Grundgesetzes nicht auf einem strikten Trennungssystem französischer oder us-amerikanischer Provenienz. 
produktiven Klerikalisierung ${ }^{(699}$ des bioethischen Diskurses und darf in ihrer negativen symbolischen Wirkung für die Integration der religiös pluralistischen deutschen Gesellschaft nicht unterschätzt werden. ${ }^{100}$

Die weitergehende Frage, ob der DER seinen Stellungnahmen und Empfehlungen explizit religiös-weltanschauliche Begründungen zugrunde legen darf, ob diesen also das Neutralitätsgebot im Sinne einer rechtlichen Pflicht zum öffentlichen Vernunftgebrauch trifft, soll im Zusammenhang mit der dritten und letzten Rechtfertigungsebene diskutiert werden.

\section{3 Zivilgesellschaft}

Für die Zivilgesellschaft, also das Ensemble der gemeinhin privat genannten Akteure, ${ }^{101}$ kann die Neutralitätspflicht des Staates schon begrifflich nicht gelten. Die Neutralitätspflicht trifft den Staat als denjenigen, den die Grundrechte verpflichten. Bürger und Religionsgemeinschaften dürfen vom Staat Neutralität verlangen, nicht aber der Staat vom Bürger. Im Gegenteil: Die Religionsfreiheit und die Kommunikationsgrundrechte sichern den Bürgern, Verbänden und Religionsgemeinschaften einen Freiraum, in dem sie sich als ethische oder gläubige Personen, in den Grenzen der allgemeinen Gesetze, ${ }^{102}$ auch öffentlich entfalten können. Interventionen religiöser Akteure zu bioethischen Streifragen durch öffentliche Stellungnahmen, politische Aktionen und durch die Mitwirkung an privat organisierten bioethischen Beratungsgremien bewegen sich vornehmlich in diesem staatsfernen Bereich der Zivilgesellschaft. Beispiele für bioethische Beratungsgremien auf dieser Ebene bilden krankenhausinterne Klinische Ethik-Komitees (KEK) und EthikKonsile, die sich organisationsintern mit Einzelfällen beschäftigen, die

99 Graf (2006), 85.

100 Vgl. dazu Graf (2009), 85 f. sowie aus politikwissenschaftlicher Perspektive Willems, Ulrich (2004), Weltanschaulich neutraler Staat, christlich-abendländische Kultur und Laizismus. Zu Struktur und Konsequenzen aktueller religionspolitischer Konflikte in der Bundesrepublik, in: Walther, Manfred (Hg.), Religion und Politik. Zur Theorie und Praxis des theologisch-politischen Komplexes, BadenBaden.

101 Der Begriff geht auf Gramsci zurück, vgl. Gramsci, Antonio (1996), Gefängnishefte, Bd. 7, Heft 12-15, Heft 12, \1, 1502.

$102 \mathrm{Zu}$ den Anforderungen an die Rechts- und Verfassungstreue von Religionsgemeinschaften vgl. Walter (2008), 1076. 
ethische Fragen aufwerfen und häufig mit Problemen der Kommunikation zwischen Ärzten, Pflegepersonal, Seelsorgern, Patienten und Angehörigen verbunden sind. ${ }^{103}$

Wie eingangs festgestellt, ist die religiös-weltanschauliche Neutralität des Staates im Kontext zivilgesellschaftlichen Handelns religiöser Akteure nicht berührt, da der Staat und nicht die religiösen Akteure durch das Neutralitätsgebot verpflichtet werden. Den Versuch einer Übertragung des Neutralitätsgebots auf die Beziehungen zwischen Bürgern, privaten Verbänden und Religionsgemeinschaften kann man in Ansätzen erblicken, die die normativen Implikationen einer Pflicht zum öffentlichen Vernunftgebrauch (John Rawls) ${ }^{104}$, für jeden Staatsbürger, also auch die religiösen Akteure betonen. Aus einer juristischen Perspektive hat die Debatte über die Reichweite der Pflicht zum öffentlichen Vernunftgebrauch, die sich an einigen neueren Stellungnahmen von Jürgen Habermas entzündet hat, ${ }^{105}$ allerdings kaum Relevanz, da die rechtliche Antwort eindeutig ist: Selbstverständlich dürfen Kirchen, Religionsgemeinschaften und Neue religiöse Bewegungen sowie - erst recht - der einzelne Gläubige auch außerhalb ihrer Gemeinden unter dem Schutz der Religions- und Meinungsfreiheit in den „wilden“ öffentlichen politischen Diskurs mit ausschließlich theologisch-religiös begründeten Stellungnahmen eingreifen. Rawls hat die Pflicht zum öffentlichen Vernunftgebrauch aus gutem Grund als moralische und nicht als rechtliche Pflicht konstruiert: „This duty, like other political rights and duties, is an intrinsically moral duty. I emphasize that it is not a legal duty, for in that case it would be incompatible with freedom of speech." "106 Eine rechtliche Verpflichtung der Bürger zum öffentlichen Vernunftgebrauch auf der Ebe-

103 Dazu Wagner, Elke (2008), Der Arzt und seine Kritiker. Zur Aktivierung authentischer Publika im Krankenhaus, in: Saake, Irmhild/Vogd, Werner (Hg.), Moderne Mythen der Medizin. Studien zur organisierten Krankenbehandlung, Wiesbaden, 265-284.

104 Rawls (2005), 312 ff.; Rawls, John (Hg.) (2002), The Law of Peoples with „The Idea of Public Reason Revisited“", 4. Auflage, Cambridge, MA, 132 ff.; dazu Habermas (2005), 123 ff.; vgl. auch das ähnliche Prinzip ,säkularer Rechtfertigung“ (principle of secular rationale) bei Audi, Robert (2000), Religious Commitment and Secular Reason, Cambridge, MA, $86 \mathrm{ff}$.

105 Zur Kritik an Habermas vgl. D’Arcais, Paolo Flores (2007), Elf Thesen zu Habermas. Die Weltreligionen sind mächtig genug. Deshalb ist es ein Fehler, wenn Philosophen sie als Sinn-Ressource der Demokratie feiern, in: Die Zeit 48, 22.11., 53 und die (überzeugende) Antwort von Habermas in Habermas (2007).

106 Rawls (Hg.) (2002), 136. 
ne der Zivilgesellschaft bedeutete nicht nur eine „säkularistische Überverallgemeinerung ${ }^{\text {‘107}}$, sie würde vielmehr mit dem Prinzip des freedom of speech zugleich die Grundlagen des politischen Liberalismus selbst beseitigen. Es kann also nur darum gehen, ob es ein moralisches Gebot der staatsbürgerlichen Vernunft gibt, das religiösen Akteuren nahe legt, auch in den bioethischen Diskursen der Zivilgesellschaft, ihre Gründe in einer säkularen Sprache zu formulieren, die prinzipiell allen Beteiligten zugänglich ist. Umgekehrt kann auch die von Habermas geforderte Bereitschaft der staatlich-politischen Akteure, religiös formulierte Gründe auf ihren vernünftigen säkularen Gehalt zu prüfen und gegebenenfalls zu übersetzen, nicht als eine rechtliche Verpflichtung betrachtet werden. ${ }^{108}$ Die Reichweite der moralischen Pflicht zum öffentlichen Vernunftgebrauch ist hier nicht weiter zu diskutieren. ${ }^{109}$

Für eine rechtliche Theorie der religiös-weltanschaulichen Neutralität des Staates besteht der Vorzug der Konzeption von Habermas darin, dass er deutlicher als andere liberale Philosophen zwischen der staatlichen Ebene und der Ebene der Zivilgesellschaft differenziert: „Wir müssen allerdings die institutionalisierten Deliberations- und Entscheidungsprozesse auf der Ebene der Parlamente, Gerichte, Ministerien und Verwaltungsbehörden klar vom informellen Engagement der Bürger in Zivilgesellschaft und politischer Öffentlichkeit unterscheiden." ${ }^{110}$ Für die hier zu diskutierende rechtliche Reichweite des Neutralitätsgebots kommt es allein auf die von Habermas betonten staatlich-institutionellen Schwellen ,zwischen der ,wilden“ politischen Öffentlichkeit und den staatlichen Körperschaften an“, deren Funktion es ist Filter zu bilden, „die aus dem Stimmengewirr der öffentlichen Kommunikationsbeiträge nur die säkularen Beiträge durchlassen. “111 Die abschließend zu beantwortende Frage ist, wo diese Schwelle im hier entwickelten Modell der Rechtfertigungskontexte bioethischer Beratungsgremien verläuft.

107 Habermas (2005), 134.

108 Gerade die Formulierung, dass der demokratische Staat nicht wissen könne, ob sich die Gesellschaft nicht von „Ressourcen der Sinn- und Identitätsstiftung“ abschneide, wenn sie die Kommunikation im öffentlichen Raum nicht für religiöse Äußerungen offen halte, siehe Habermas (2007), 1444, ist auf Kritik gestoBen.

109 Dazu Graf (2006), 66 sowie die in Fn. 102 genannten Beiträge.

110 Habermas (2007), 1444.

111 Habermas (2005), 137. 
Diese Grenze muss dort gezogen werden, wo sich die Rechtfertigungsanforderungen nicht an private Akteure der Zivilgesellschaft als private Akteure richten, sondern an Institutionen, die im gesetzlichen Auftrag öffentliche Aufgaben erfüllen und organisatorisch der öffentlichen Staatsverwaltung zugeordnet werden können (vgl. Abbildung). Der DER liegt jenseits dieser Schwelle, da er als öffentlich-rechtlich verfasstes Gremium privilegierter Beratung in gesetzlichem Auftrag öffentliche Aufgaben erfüllt und staatsorganisatorisch Teil der unmittelbaren Staatsverwaltung ist. Der DER darf nicht deshalb, weil er unter anderem die Aufgabe hat, die Diskussion in der Gesellschaft zu fördern, so behandelt werden als sei er die Zivilgesellschaft oder als würde er diese abbilden. Indem er die ,wilde politische Öffentlichkeit“ im Wege der „Deliberation von oben“ durch Institutionalisierung bewusst organisiert und steuert, unterscheidet er sich signifikant von den pluralen, netzwerkartigen und unberechenbaren Formen der „Deliberation von unten“. Damit soll keineswegs behauptet werden, dass der DER die Öffentlichkeit von vorneherein in eine bestimmte Richtung gezielt beeinflussen möchte. Es kann jedoch nicht bestritten werden, dass seinen veröffentlichten Stellungnahmen eine privilegierte Bedeutung für die politischen Prozesse der Begründung bioethischer Normen zukommt. Als bedeutender staatlicher biopolitischer Akteur erweist sich der DER zudem bereits durch die Auswahl seiner Themen.

Abschließend kann festgestellt werden, dass das Neutralitätsgebot den DER auch in seiner materiell-inhaltlichen Dimension verpflichtet. Das verfassungsrechtliche Gebot ethischer Neutralität des Staates steht explizit religiös-weltanschaulichen Begründungen von Stellungnahmen des DER entgegen. Die empirische Auswertung der Wortprotokolle und der veröffentlichten Stellungnahmen des Nationalen Ethikrats (NER) ${ }^{112}$ zeigt, dass die Religionsvertreter in der Praxis des Ethikrats bisher auf einen Gebrauch religiöser und theologischer Sprache verzichtet oder diese nur unter einem Übersetzungsvorbehalt in die Diskussion eingeführt haben. ${ }^{113} \mathrm{Ob}$ dies Ausdruck einer reflexiven, post-säkularen Vernunft

112 Der NER wurde durch den DER abgelöst.

113 Instruktiv Voigt (2008), 260 ff.; Voigt betont jedoch am Beispiel der von Religionsvertretern geschriebenen Sondervoten, dass auch dort, wo nicht mit expliziten religiösen Begriffen gearbeitet würde, hinter den Voten ,,theologische Reflexion“ stecke. Vgl. auch Bogner (2009), der das empirische Material stärker auf das Problem der Marginalisierung der Fachethik hin untersucht. 
ist $^{114}$ oder eher das Ergebnis einer Eigendynamik, die die kommunikative Praxis in interdisziplinären Kommissionen gleichsam hinter dem Rücken der Akteure entfaltet, ist eine Frage, die der weiteren soziologischen Aufklärung bedarf.

\section{Fazit}

Aus juristischer Perspektive stellt der Einfluss religiöser Akteure in bioethischen Beratungsgremien ein Problem der demokratischen und rechtsstaatlichen Legitimation der Rechtfertigung von Normen und Entscheidungen im biopolitischen Diskurs dar: Religion in bioethischen Diskursen ist prima facie rechtsstaatlich bedenklich. Die juristische Bewertung des Einflusses der Religion auf bioethische Beratungsgremien erfordert eine differenzierte Betrachtung des unübersichtlichen Feldes der Bioethik-Beratung. Die Rechtsanwendung durch Ethikkommissionen im Verwaltungsverfahren, die staatlich-institutionalisierte Politikberatung durch den DER und die zivilgesellschaftlichen Formen der Bioethik-Beratung bilden unterschiedliche Kontexte der Rechtfertigung, die dem Neutralitätsgebot unterschiedliche Bedeutung zuweisen. Der religionsverfassungsrechtliche Grundsatz der religiös-weltanschaulichen Neutralität des Staates greift überall dort ein, wo Beratungsgremien öffentliche Aufgaben als Teil der Staatsverwaltung erfüllen. Das Neutralitätsgebot betrifft damit nicht nur die Ethikkommissionen im Verwaltungsverfahren, sondern auch den DER. Die Intensität der Anforderungen an die rechtsstaatliche Legitimation solcher Gremien und die Reichweite der materiell-inhaltlichen Neutralitätspflicht hängen aber auch davon ab, in wieweit ihre Stellungnahmen faktischen oder rechtlichen Einfluss auf individuelle Grundrechtspositionen haben. Vor diesem Hintergrund scheint die tatsächliche Praxis der Besetzung von bioethischen Beratungsgremien mit Religionsvertretern der beiden großen christlichen Kirchen nicht immer zweckmäßig und mit Blick auf das Neutralitätsgebot zum Teil auch rechtsstaatlich bedenklich. Auf der nicht-staatlichen Ebene der Zivilgesellschaft ist der Einfluss der Religion auf bioethische Diskurse, in den Grenzen der allgemeinen Gesetze, nicht nur rechtlich unproblematisch, sondern sogar grundrechtlich geschützt. Das Gebot des öffentlichen Vernunftgebrauchs (Rawls) ist moralischer, nicht rechtlicher Natur.

114 Dazu Voigt (2008), 267 f. 
Abbildung: Bioethische Beratungsgremien - Rechtfertigungskontexte

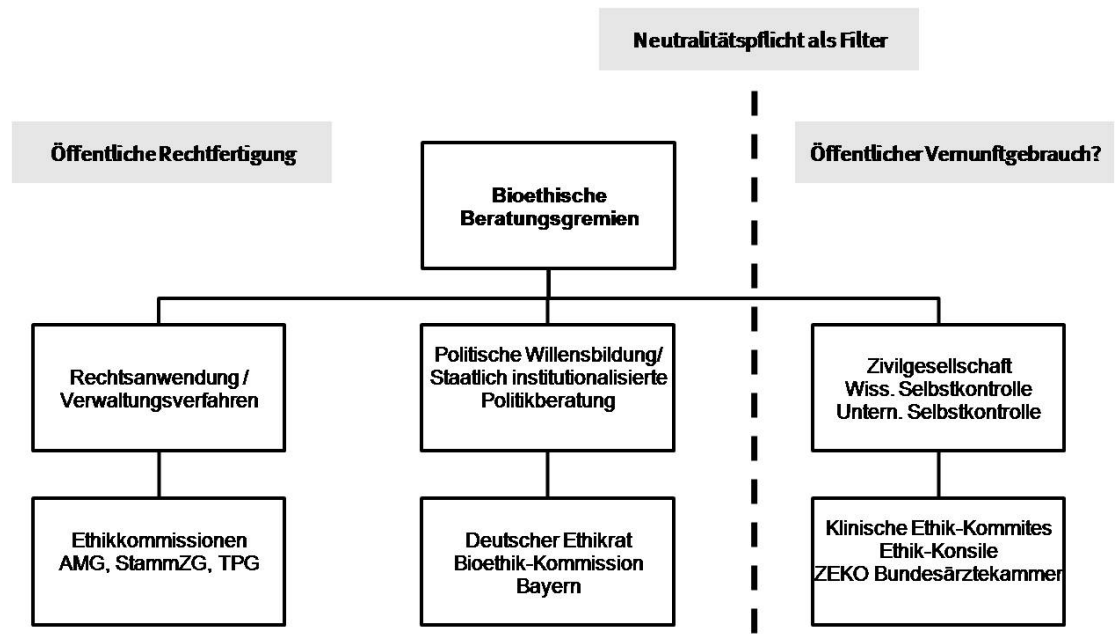

\section{Literatur}

After Secularisation (2006), The Hedgehog Review 8.

Albers, Marion (2003), Die Institutionalisierung von Ethik-Kommissionen: Zur Renaissance der Ethik im Recht, in: Kritische Vierteljahresschrift für Gesetzgebung und Rechtswissenschaft 86, 419-436.

Audi, Robert (2000), Religious Commitment and Secular Reason, Cambridge.

Beck, Ulrich (2008), Der eigene Gott. Von der Friedensfähigkeit und dem Gewaltpotential der Religionen, Frankfurt a. M.

Birnbacher, Dieter (2003), „Hochrangigkeit“ im Stammzellgesetz - Ein Kommentar aus ethischer Sicht, in: Honnefelder, Ludger/Streffer, Christian (Hg.), Jahrbuch für Wissenschaft und Ethik, Bd. 8, Berlin, New York, 353-359.

Böckenförde, Ernst-Wolfgang (2006), Die Entstehung des Staates als Vorgang der Säkularisation, in: ders. (Hg.), Recht, Staat, Freiheit. Studien zur Rechtsphilosophie, Staatstheorie und Verfassungsgeschichte, Frankfurt a. M., 92-114.

Bogner, Alexander (2009), Ethisierung und die Marginalisierung der Ethik. Zur Mikropolitik des Wissens in Ethikräten, in: Soziale Welt 60, 119-137.

Bourdieu, Pierre (2009), Religion, Konstanz.

Brugger, Winfried (2007), Von Feindschaft über Anerkennung zur Identifikation. Staat-Kirche-Modelle und ihr Verhältnis zur Religionsfreiheit, in: Joas, 
Hans/Wiegandt, Klaus (Hg.), Säkularisierung und die Weltreligionen, Frankfurt a. M., 253-283.

Casanova, José (1994), Public Religions in the Modern World, Chicago.

Casanova, José (2004), Religion und Öffentlichkeit. Ein Ost-/Westvergleich, in: Gabriel, Karl/Reuter, Hans-Richard (Hg.), Religion und Gesellschaft. Texte zur Religionssoziologie, Paderborn, 271-293.

Casanova, José (2006), Secularization Revisited: A Reply to Talal Asad, in: Scott, David/Hirschkind, Charles (Hg.), Powers of the Secular Modern: Talal Asad and His Interlocutors, Stanford, 12-30.

Casanova, José (2008), Public Religions Revisited, in: Große Kracht, HermannJosef (Hg.), Christentum und Solidarität. Bestandsaufnahmen zu Sozialethik und Religionssoziologie, Paderborn, 313-338.

Cover, Robert M. (1983), The Supreme Court. 1982 Term. Foreword: Nomos and Narrative, in: Harvard Law Review 97, 4-68.

Czermak, Gerhard/Hilgendorf, Eric (2008), Religions- und Weltanschauungsrecht. Eine Einführung, Berlin.

D'Arcais, Paolo Flores (2007), Elf Thesen zu Habermas. Die Weltreligionen sind mächtig genug. Deshalb ist es ein Fehler, wenn Philosophen sie als Sinn-Ressource der Demokratie feiern, in: Die Zeit 48, 22.11., 53.

Deutsch, Erwin (2006), Das neue Bild der Ethikkommission, in: Medizinrecht 24, 411-416.

Fateh-Moghadam, Bijan/Atzeni, Gina (2009), Ethisch vertretbar im Sinne des Gesetzes. Zum Verhältnis von Ethik und Recht am Beispiel der Praxis von ForschungsEthikkommisionen, in: Vöneky, Silja u. a. (Hg.), Legitimation ethischer Entscheidungen im Recht. Interdisziplinäre Untersuchungen, Berlin, Heidelberg, 114-143.

Forst, Rainer (2004), Kontexte der Gerechtigkeit. Politische Philosophie jenseits von Liberalismus und Kommunitarismus, Frankfurt a. M.

Forst, Rainer (2007), Das Recht auf Rechtfertigung. Elemente einer konstruktivistischen Theorie der Gerechtigkeit, Frankfurt a. M.

Graf, Friedrich Wilhelm (2005), Die Wiederkehr der Götter. Religion in der modernen Kultur, München.

Graf, Friedrich Wilhelm (2006), Moses Vermächtnis. Über göttliche und menschliche Gesetze, München.

Graf, Friedrich Wilhelm (2009), Missbrauchte Götter. Zum Menschenbilderstreit in der Moderne, München.

Gramsci, Antonio (1996), Gefängnishefte, Bd. 7, Heft 12-15.

Große Kracht, Hermann-Josef (2004), Religionen zwischen Säkularisierung und Entprivatisierung: José Casanovas These vom Wiedererstarken öffentlicher Religionen in der späten Moderne. Einführung, in: Gabriel, Karl/Reuter, Hans-Richard 
(Hg.), Religion und Gesellschaft. Texte zur Religionssoziologie, Paderborn, 269270.

Gutmann, Thomas (2008), Christliche Imprägnierung des Strafgesetzbuchs?, in: Dreier, Horst/Hilgendorf, Eric (Hg.), Kulturelle Identität als Grund und Grenze des Rechts. Akten der IVR-Tagung vom 28.-30. September 2006 in Würzburg, Stuttgart, 295-313.

Habermas, Jürgen (1983), Diskursethik - Notizen zu einem Begründungsprogramm, in: Habermas, Jürgen (Hg.), Moralbewußtsein und kommunikatives Handeln, Frankfurt a. M., 53-126.

Habermas, Jürgen (1998), Faktizität und Geltung. Beiträge zur Diskurstheorie des Rechts und des demokratischen Rechtsstaats, Frankfurt a. M.

Habermas, Jürgen (2005), Zwischen Naturalismus und Religion. Philosophische Aufsätze, Frankfurt a. M.

Habermas, Jürgen (2007), Die öffentliche Stimme der Religion. Säkularer Staat und Glaubenspluralismus, in: Blätter für deutsche und internationale Politik, 1441-1446.

Heinig, Hans Michael (2009), Verschärfung der oder Abschied von der Neutralität? Zwei verfehlte Alternativen in der Debatte um den herkömmlichen Grundsatz religiös-weltanschaulicher Neutralität, in: Juristenzeitung, 1136-1140.

Heinig, Hans Michael/Walter, Christian (Hg.) (2007), Staatskirchenrecht oder Religionsverfassungsrecht? Ein begriffspolitischer Grundsatzstreit, Tübingen.

Hochgeschwender, Michael (2007), Amerikanische Religion. Evangelikalismus, Pfingstlertum und Fundamentalismus, Frankfurt a. M.

Hofmann, Hasso (2003), Recht, Politik und Religion, in: Juristenzeitung 58, $377-$ 385.

Höver, Gerhard/Eibach, Ulrich (2003), Die aktuelle Biomedizin aus der Sicht der christlichen Kirchen, in: Schicktanz, Silke/Tannert, Christof/Wiedemann, Peter (Hg.), Kulturelle Aspekte der Biomedizin. Bioethik, Religionen und Alltagsperspektiven, Frankfurt a. M., 16-55.

Huster, Stefan (2002), Die ethische Neutralität des Staates. Eine liberale Interpretation der Verfassung, Tübingen.

Ilkilic, Ilhan (2003), Die aktuelle Biomedizin aus der Sicht des Islam, in: Schicktanz, Silke/Tannert, Christof/Wiedemann, Peter (Hg.), Kulturelle Aspekte der Biomedizin. Bioethik, Religionen und Alltagsperspektiven, Frankfurt a. M., 56-83.

Jarass, Hans D./Pieroth, Bodo (2009), Grundgesetz für die Bundesrepublik Deutschland. Kommentar, 10. Auflage, München.

Knoblauch, Hubert (2009), Populäre Religion. Auf dem Weg in eine spirituelle Gesellschaft, Frankfurt a. M.

Kokott, Juliane (2009), Art. 4, in: Sachs, Michael (Hg.), Grundgesetz. Kommentar, München, 237-274. 
Kraus, Wolfgang (2003), Ein Vergleich der christlichen, islamischen und jüdischen Perspektiven, in: Schicktanz, Silke/Tannert, Christof/Wiedemann, Peter (Hg.), Kulturelle Aspekte der Biomedizin. Bioethik, Religionen und Alltagsperspektiven, Frankfurt a. M., 107-131.

Lübbe, Hermann (2003), Säkularisierung. Geschichte eines ideenpolitischen Begriffs, 3. Auflage, Freiburg.

Luckmann, Thomas (1991), Die unsichtbare Religion, Frankfurt a. M.

Luhmann, Niklas (1998), Die Gesellschaft der Gesellschaft, Frankfurt a. M.

Luhmann, Niklas (2002), Die Religion der Gesellschaft, Frankfurt a. M.

Merkel, Reinhard (2007), Der Schwangerschaftsabbruch, in: Roxin, Claus/Schroth, Ulrich (Hg.), Handbuch des Medizinstrafrechts, 3. Auflage, Stuttgart, 145-231.

Morlok, Martin (2004), Art. 4 GG, in: Dreier, Horst (Hg.), Grundgesetz. Kommentar. Präambel, Artikel 1-19, Bd. 1, Tübingen, 484-549.

Morlok, Martin (2008), Art. 140 GG, in: Dreier, Horst (Hg.), Grundgesetz. Kommentar. Artikel 83-146, Bd. 3, München, 1512-1669.

Nordmann, Yves/Birnbaum, Michel (2003), Die aktuelle Biomedizin aus der Sicht des Judentums, in: Schicktanz, Silke/Tannert, Christof/Wiedemann, Peter (Hg.), Kulturelle Aspekte der Biomedizin. Bioethik, Religionen und Alltagsperspektiven, Frankfurt a. M., 84-105.

Pollack, Detlef (2009), Rückkehr des Religiösen? Studien zum religiösen Wandel in Deutschland und Europa II, Tübingen.

Rawls, John (1997), Die Idee des politischen Liberalismus. Aufsätze 1978-1989, Frankfurt a. M.

Rawls, John (Hg.) (2002), The Law of Peoples with "The Idea of Public Reason Revisited", 4. Auflage, Cambridge, MA.

Rawls, John (2005), Politischer Liberalismus, Frankfurt a. M.

Roellecke, Gerd (2004), Die Entkoppelung von Recht und Religion, in: Juristenzeitung 59, 105-110.

Schicktanz, Silke/Tannert, Christof/Wiedemann, Peter (Hg.) (2003), Kulturelle Aspekte der Biomedizin. Bioethik, Religionen und Alltagsperspektiven, Frankfurt a. M.

Schleissing, Stephan/Zichy, Michael (2009), Religion in bioethischen Diskursen. Internationale und interreligiöse Perspektiven. Rückblick auf ein Symposium vom 18. bis 19. Februar 2009 in München, in: Zeitschrift für Evangelische Ethik 3, 215-219.

Schmidt-Aßmann, Eberhard (2001), Grundrechtspositionen und Legitimationsfragen im öffentlichen Gesundheitswesen. Verfassungsrechtliche Anforderungen an Entscheidungsgremien in der gesetzlichen Krankenversicherung und im Transplantationswesen, Berlin, New York. 
Schroth, Ulrich (2007), Stammzellenforschung und Präimplantationsdiagnostik aus juristischer und ethischer Sicht, in: Roxin, Claus/ders. (Hg.), Handbuch des Medizinstrafrechts, 3. Auflage, Stuttgart, 435-461.

Sutter, Barbara/Maasen, Sabine (2010), "Bürgergesellschaft". Der verdeckte Paternalismus eines politischen Programms, in: Fateh-Moghadam, Bijan/Sellmaier, Stephan/Vossenkuhl, Wilhelm (Hg.), Grenzen des Paternalismus, Stuttgart, 318-340.

Taupitz, Jochen (2003), Ethikkommissionen in der Politik: Bleibt die Ethik auf der Strecke?, in: Juristenzeitung, 815-821.

Taylor, Charles (2009), Ein säkulares Zeitalter, Frankfurt a. M.

Voigt, Friedemann (2008), Religion und Religionsvertreter in ethischen Diskursen und Kommissionen, in: Zichy, Michael/Grimm, Herwig (Hg.), Praxis in der Ethik. Zur Methodenreflexion in der anwendungsorientierten Moralphilosophie, Berlin, New York, 249-273.

Wagner, Elke (2008), Der Arzt und seine Kritiker. Zur Aktivierung authentischer Publika im Krankenhaus, in: Saake, Irmhild/Vogd, Werner (Hg.), Moderne Mythen der Medizin. Studien zur organisierten Krankenbehandlung, Wiesbaden, 265-284.

Walter, Christian (2006), Religionsverfassungsrecht, Tübingen.

Walter, Christian (2008), Religiöse Freiheit als Gefahr? Eine Gegenrede, in: Das Deutsche Verwaltungsblatt, 1073-1080.

Weber, Max/Kaesler, Dirk (2004), Die protestantische Ethik und der Geist des Kapitalismus, München.

Willems, Ulrich (2004), Weltanschaulich neutraler Staat, christlich-abendländische Kultur und Laizismus. Zu Struktur und Konsequenzen aktueller religionspolitischer Konflikte in der Bundesrepublik, in: Walther, Manfred (Hg.), Religion und Politik. Zur Theorie und Praxis des theologisch-politischen Komplexes, Baden-Baden.

Wölk, Florian (2002), Zwischen ethischer Beratung und rechtlicher Kontrolle Aufgaben und Funktionswandel der Ethikkommissionen in der medizinischen Forschung am Menschen, in: Ethik in der Medizin 14, 252-269. 